\title{
Global existence for a nonstandard viscous Cahn-Hilliard system with dynamic boundary condition*
}

\author{
Pierluigi Colli ${ }^{(1)}$ \\ e-mail: pierluigi.colli@unipv.it \\ Gianni Gilardi ${ }^{(1)}$ \\ e-mail: gianni.gilardi@unipv.it \\ Jürgen Sprekels ${ }^{(2)}$ \\ e-mail: sprekels@wias-berlin.de \\ (1) Dipartimento di Matematica "F. Casorati", Università di Pavia \\ via Ferrata 5, 27100 Pavia, Italy \\ (2) Weierstrass Institute for Applied Analysis and Stochastics \\ Mohrenstraße 39, 10117 Berlin, Germany, and \\ Department of Mathematics, Humboldt-Universität zu Berlin \\ Unter den Linden 6, 10099 Berlin. Germany
}

\begin{abstract}
In this paper, we study a model for phase segregation taking place in a spatial domain that was introduced by Podio-Guidugli in Ric. Mat. 55 (2006), pp. 105-118. The model consists of a strongly coupled system of nonlinear parabolic differential equations, in which products between the unknown functions and their time derivatives occur that are difficult to handle analytically. In contrast to the existing literature about this PDE system, we consider here a dynamic boundary condition involving the Laplace-Beltrami operator for the order parameter. This boundary condition models an additional nonconserving phase transition occurring on the surface of the domain. Different well-posedness results are shown, depending on the smoothness properties of the involved bulk and surface free energies.
\end{abstract}

Key words: viscous Cahn-Hilliard system, phase field model, dynamic boundary conditions, well-posedness of solutions

AMS (MOS) Subject Classification: 35K61, 35A05, 35B40, 74A15.

*This work received a partial support from the GNAMPA (Gruppo Nazionale per l'Analisi Matematica, la Probabilità e loro Applicazioni) of INDAM (Istituto Nazionale di Alta Matematica) and the IMATI C.N.R. Pavia for PC and GG. 


\section{Introduction}

Let $\Omega \subset \mathbb{R}^{3}$ be a bounded and connected open set with a smooth boundary $\Gamma$, as well as $Q:=\Omega \times(0, T)$ and $\Sigma:=\Gamma \times(0, T)$. We denote by $\partial_{\mathbf{n}}, \nabla_{\Gamma}, \Delta_{\Gamma}$, the outward normal derivative, the tangential gradient, and the Laplace-Beltrami operator on $\Gamma$, in this order. We then consider the initial-boundary value problem

$$
\begin{aligned}
& (1+2 g(\rho)) \partial_{t} \mu+\mu g^{\prime}(\rho) \partial_{t} \rho-\Delta \mu=0 \quad \text { in } Q, \\
& \partial_{\mathbf{n}} \mu=0 \quad \text { on } \Sigma, \\
& \partial_{t} \rho-\Delta \rho+\xi+\pi(\rho)=\mu g^{\prime}(\rho) \quad \text { in } Q, \\
& \xi \in \beta(\rho) \quad \text { a.e. in } Q, \\
& \partial_{\mathbf{n}} \rho+\partial_{t} \rho_{\Gamma}+\xi_{\Gamma}+\pi_{\Gamma}\left(\rho_{\Gamma}\right)-\Delta_{\Gamma} \rho_{\Gamma}=u_{\Gamma}, \quad \rho_{\Gamma}=\rho_{\mid \Sigma}, \quad \text { on } \Sigma, \\
& \xi_{\Gamma} \in \beta_{\Gamma}\left(\rho_{\Gamma}\right) \quad \text { a.e. on } \Sigma, \\
& \mu(0)=\mu_{0}, \quad \rho(0)=\rho_{0}, \quad \text { in } \Omega, \quad \rho_{\Gamma}(0)=\rho_{0 \mid \Gamma} \quad \text { on } \Gamma .
\end{aligned}
$$

We point out that $\beta$ and $\beta_{\Gamma}$ denote two maximal monotone graphs, with domains $D(\beta) \supseteq$ $D\left(\beta_{\Gamma}\right)$ that are intervals containing $0 ; \beta$ and $\beta_{\Gamma}$ fulfill suitable properties (cf. the later (A3) and (A7) ) about their values and growths; $\pi$ and $\pi_{\Gamma}$ denote two Lipschitz continuous perturbations; $g$ is a smooth nonnegative and concave function defined on $D(\beta) ; u_{\Gamma}$ is a boundary datum.

The system (1.1)-(1.7) is related to a model for phase segregation through atom rearrangement on a lattice that has been proposed in [44]. This model (see also [14] for a detailed derivation) is a modification of the Fried-Gurtin approach to phase segregation processes (cf. [31,38]). The order parameter $\rho$, which in many cases represents the (normalized) density of one of the phases, and the chemical potential $\mu$ are the unknowns of the system.

Let us note at once that, in our present contribution, the Neumann homogeneous boundary condition (1.2) is considered for $\mu$, while, differently from previous analyses $[11,14-20]$, the dynamic boundary condition (1.5) is assumed for $\rho_{\Gamma}$, the trace of $\rho$ on the boundary.

The approach by Podio-Guidugli [44] is based on a local free energy density (in the bulk) of the form

$$
\psi(\rho, \nabla \rho, \mu)=-\mu \rho+W(\rho)+\frac{1}{2}|\nabla \rho|^{2},
$$

where $W$ is a double-well potential, whose derivative (in the differentiable case) plays as the sum $\beta+\pi$ in (1.3) (see also (1.4)). By (1.8), one arrives at the evolutionary system

$$
\begin{aligned}
& 2 \rho \partial_{t} \mu+\mu \partial_{t} \rho-\Delta \mu=0 \\
& -\Delta \rho+W^{\prime}(\rho)=\mu .
\end{aligned}
$$

The above equations are assumed to hold in $Q$ and must be complemented with boundary and initial conditions. The typical example for a smooth double-well potential $W$ is

$$
W(r)=\frac{1}{4}\left(r^{2}-1\right)^{2}, \quad r \in \mathbb{R}
$$


while another smooth potential, but defined on a bounded interval, is given by

$$
W(r)=(1+r) \ln (1+r)+(1-r) \ln (1-r)-c r^{2}, \quad r \in(-1,1),
$$

where the coefficient $c$ is taken greater than 1 in order that $W$ be nonconvex. The potentials (1.11) and (1.12) are usually referred to as the classical regular and the logarithmic double-well potential, respectively. Observe that the derivative of the logarithmic potential becomes singular at \pm 1 . However, one can consider nondifferentiable potentials, where an important example is given by the so-called double-obstacle potential

$$
W(r)=I_{[-1,1]}(r)-c r^{2}, \quad r \in \mathbb{R},
$$

where $c>0$ is a positive constant and $I_{[-1,1]}: \mathbb{R} \rightarrow[0,+\infty]$ denotes the indicator function of $[-1,1]$, i.e., we have $I_{[-1,1]}(r)=0$ if $|r| \leq 1$ and $I_{[-1,1]}(r)=+\infty$ otherwise. In this case, the order parameter is subjected to the unilateral constraint $|\rho| \leq 1$, and (1.10) should be read as a differential inclusion where $W^{\prime}(\rho)=\beta(\rho)+\pi(\rho)$, with $\beta$ representing the subdifferential $\partial I_{[-1,1]}$ of $I_{[-1,1]}$ and $\pi(\rho)=-2 c \rho$.

The system (1.9)-(1.10) is a variation of the Cahn-Hilliard system originally introduced in [4] and first studied mathematically in the seminal paper [30] (for an updated list of references on the Cahn-Hilliard system, see [39]). An initial-boundary value problem for (1.9)-(1.10) is in general ill-posed: indeed, as it was pointed out in [17], when assuming Neumann homogeneous boundary conditions for both $\rho$ and $\mu$, the related problem may have infinitely many smooth and even nonsmooth solutions. Then, two small regularizing parameters $\varepsilon>0$ and $\delta>0$ were considered in [14], which led to the regularized model equations

$$
\begin{aligned}
& (\varepsilon+2 \rho) \partial_{t} \mu+\mu \partial_{t} \rho-\Delta \mu=0, \\
& \delta \partial_{t} \rho-\Delta \rho+F^{\prime}(\rho)=\mu
\end{aligned}
$$

The system (1.14)-(1.15) constitutes a modification of the so-called viscous Cahn-Hilliard system (see [43] and the recent papers [6,22,24] along with their references). We point out that (1.14)-(1.15) was analyzed, in the case of no-flux boundary conditions for both $\mu$ and $\rho$, in the papers $[14,16,18,20]$ concerning well-posedness, regularity, asymptotics as $\varepsilon \searrow 0$, and optimal control. Later, the local free energy density (1.8) was generalized to the form

$$
\psi(\rho, \nabla \rho, \mu)=-\mu g(\rho)+W(\rho)+\frac{1}{2}|\nabla \rho|^{2},
$$

with a function $g$ having suitable properties. If one puts, without loss of generality, $\varepsilon=\delta=1$, then one obtains the more general system

$$
\begin{aligned}
& (1+2 g(\rho)) \partial_{t} \mu+\mu g^{\prime}(\rho) \partial_{t} \rho-\Delta \mu=0, \\
& \partial_{t} \rho-\Delta \rho+W^{\prime}(\rho)=\mu g^{\prime}(\rho),
\end{aligned}
$$

which was investigated in the contributions $[11,15,17,19]$, still for no-flux boundary conditions, also from the side of the numerical approximation. The related phase relaxation system (in which the diffusive term $-\Delta \rho$ disappears from (1.18)), has been dealt with in $[12,13,21]$. We also mention the recent article [25], where a nonlocal version of (1.17)(1.18) - based on the replacement of the diffusive term of (1.18) with a nonlocal operator acting on $\rho$ - has been largely investigated. 
In the present paper, we consider a total free energy of the system which also includes a contribution on the boundary; in fact, we postulate that a phase transition phenomenon is occurring as well on the boundary, and the physical variable on the boundary is just the trace of the phase variable in the bulk. Then, we choose a total free energy functional of the form

$$
\begin{aligned}
\Psi\left[\mu(t), \rho(t), \rho_{\Gamma}(t)\right]= & \int_{\Omega}\left[-\mu g(\rho)+W(\rho)+\frac{1}{2}|\nabla \rho|^{2}\right](t) \mathrm{d} x \\
& +\int_{\Gamma}\left[-u_{\Gamma} \rho_{\Gamma}+W_{\Gamma}\left(\rho_{\Gamma}\right)+\frac{1}{2}\left|\nabla_{\Gamma} \rho_{\Gamma}\right|^{2}\right](t) \mathrm{d} \Gamma, \quad t \in[0, T],
\end{aligned}
$$

where $W_{\Gamma}$ denotes a double-well potential having more or less the same behavior as $W$, and $u_{\Gamma}$ is a source term that may exert a (boundary) control on the system. From this expression of the total free energy, one recovers the PDE system resulting in (1.1)-(1.7); in particular, we point out that the derivative or subdifferential of $W_{\Gamma}$ is expressed by the $\operatorname{sum} \beta_{\Gamma}+\pi_{\Gamma}$ in $(1.5)-(1.6)$.

Our aim here is to prove the well-posedness of (1.1)-(1.7) as well as regularity properties of the solution, like the $L^{\infty}$-boundedness of both variables $\mu$ and $\rho$, and consequently of $\rho_{\Gamma}$ on the boundary, and the so-called strict separation property, in the case when $W$ and $W_{\Gamma}$ behave like (1.12), which means to show that $\rho$ and $\rho_{\Gamma}$ are uniformly bounded away from -1 and +1 . We employ certain techniques which combine some key ideas essentially from the papers [14] and [19] together with the treatment of dynamic boundary conditions devised in [5] and exploited in other solvability studies and optimal control theories, namely, [8-10,22-24,26]. For these reasons, we often refer to the abovementioned papers in running the proofs.

About dynamic boundary conditions, let us notice that there has been a recent growing interest about the justification and the study of phase field models, as well as systems of Allen-Cahn and Cahn-Hilliard type, including dynamic boundary conditions. Without trying to be exhaustive, we mention at least the papers [7, 27, 28, 32-37, 40-42].

The paper is organized as follows: in Section 2, we formulate the relevant assumptions on the data of system (1.1)-(1.7), and we state the main results of this paper. Section 3 then brings a detailed proof of the existence result stated in Theorem 2.1, while Section 4 deals with the proofs of Theorem 2.2 and Theorem 2.4.

Throughout the paper, for a general Banach space $X$, we denote by $\|\cdot\|_{X}$ its norm and by $X^{\prime}$ its dual space. The only exemption from this convention are the norms of the $L^{p}$ spaces and of their powers, which we often denote by $\|\cdot\|_{p}$, for $1 \leq p \leq+\infty$. Moreover, we often utilize the continuity of the embedding $H^{1}(\Omega) \subset L^{p}(\Omega)$ for $1 \leq p \leq 6$ and the related Sobolev inequality

$$
\|v\|_{p} \leq C_{\Omega}\|v\|_{H^{1}(\Omega)} \quad \text { for every } v \in H^{1}(\Omega) \text { and } 1 \leq p \leq 6,
$$

where $C_{\Omega}$ depends only on $\Omega$. Notice that these embeddings are compact for $1 \leq p<6$. We also use the corresponding compactness inequality

$$
\|v\|_{4} \leq \delta\|\nabla v\|_{2}+\widetilde{C}_{\delta}\|v\|_{2} \text { for every } v \in H^{1}(\Omega) \text { and } \delta>0,
$$

where $\widetilde{C}_{\delta}$ depends only on $\Omega$ and $\delta$, and recall that the embedding $H^{2}(\Omega) \subset C^{0}(\bar{\Omega})$ is compact. Furthermore, we make repeated use of the notation

$$
Q_{t}:=\Omega \times(0, t), \quad \Sigma_{t}:=\Gamma \times(0, t), \quad \text { for } t \in(0, T],
$$


as well as of Hölder's inequality and of the elementary Young inequalities

$$
\begin{aligned}
& |a b| \leq \gamma|a|^{2}+\frac{1}{4 \gamma}|b|^{2} \quad \text { and } \quad|a b| \leq \frac{\gamma^{p}}{p}|a|^{p}+\frac{\gamma^{-q}}{q}|b|^{q}, \\
& \text { for every } a, b \in \mathbb{R}, \gamma>0, \text { and } 1<p, q<+\infty \text { with } \frac{1}{p}+\frac{1}{q}=1 .
\end{aligned}
$$

\section{General assumptions and main results}

In this section, we formulate the general assumptions for the data of the system (1.1)(1.7), and we state the main results of this paper. To begin with, we introduce some denotations. We set

$$
\begin{aligned}
& H:=L^{2}(\Omega), \quad V:=H^{1}(\Omega), \quad W:=\left\{w \in H^{2}(\Omega): \partial_{\mathbf{n}} w=0 \text { on } \Gamma\right\}, \\
& H_{\Gamma}:=L^{2}(\Gamma), \quad V_{\Gamma}:=H^{1}(\Gamma),
\end{aligned}
$$

and endow these spaces with their standard norms. Notice that we have $V \subset H \subset V^{\prime}$ and $V_{\Gamma} \subset H_{\Gamma} \subset V_{\Gamma}^{\prime}$ with dense, continuous and compact embeddings. Moreover, for every proper, convex and lower semicontinuous function $\widehat{\alpha}: \mathbb{R} \rightarrow[0,+\infty]$ satisfying $\widehat{\alpha}(0)=0$, we denote by $\alpha:=\partial \widehat{\alpha}$ its subdifferential, which is known to be a maximal monotone graph in $\mathbb{R} \times \mathbb{R}$ satisfying $0 \in \alpha(0)$. We denote its effective domain by $D(\alpha)$, and, for $r \in D(\alpha)$, by $\alpha^{\circ}(r)$ the element of $\alpha(r)$ having minimal modulus. Moreover, for any $\varepsilon>0$, we denote by $\alpha_{Y}^{\varepsilon}$ the Yosida approximation of $\alpha$ at the level $\varepsilon>0$. As is well known (see, e.g, $\left[2\right.$, p. 28]), $\alpha_{Y}^{\varepsilon}$ is a monotone and Lipschitz continuous function on $\mathbb{R}$, and we have that

$$
\left|\alpha_{Y}^{\varepsilon}(r)\right| \leq\left|\alpha^{\circ}(r)\right| \quad \forall \varepsilon>0, \quad \text { as well as } \quad \lim _{\varepsilon \searrow 0} \alpha_{Y}^{\varepsilon}(r)=\alpha^{\circ}(r), \quad \text { for all } r \in D(\beta) .
$$

Moreover, the antiderivative

$$
\widehat{\alpha}^{\varepsilon}(r):=\int_{0}^{r} \alpha_{Y}^{\varepsilon}(s) \mathrm{d} s, \quad r \in \mathbb{R},
$$

is a convex function on $\mathbb{R}$ that satisfies

$$
0 \leq \widehat{\alpha}_{Y}^{\varepsilon}(r) \leq \widehat{\alpha}(r), \quad \widehat{\alpha}_{Y}^{\varepsilon}(r) \nearrow \widehat{\alpha}(r) \text { as } \varepsilon \searrow 0, \text { for all } r \in \mathbb{R} .
$$

We make the following general assumptions:

(A1) $\quad \mu_{0} \in W, \quad \mu_{0} \geq 0$ a. e. in $\Omega, \quad \rho_{0} \in H^{2}(\Omega), \quad \rho_{0_{\Gamma}}:=\rho_{0_{\mid \Gamma}} \in H^{2}(\Gamma)$.

(A2) $u_{\Gamma} \in H^{1}\left(0, T ; H_{\Gamma}\right)$.

(A3) $\beta=\partial \widehat{\beta}, \beta_{\Gamma}=\partial \widehat{\beta}_{\Gamma}$, where $\widehat{\beta}, \widehat{\beta}_{\Gamma}: \mathbb{R} \rightarrow[0,+\infty]$ are proper, convex, and lower semicontinuous functions satisfying $\widehat{\beta}(0)=\widehat{\beta}_{\Gamma}(0)=0$.

We remark that (A3) entails that $\beta$ and $\beta_{\Gamma}$ are maximal monotone graphs in $\mathbb{R} \times \mathbb{R}$, with $0 \in \beta(0)$ and $0 \in \beta_{\Gamma}(0)$, whose Yosida approximations $\beta_{Y}^{\varepsilon}$ and $\beta_{\Gamma_{Y}}^{\varepsilon}$ satisfy the conditions (2.1)-(2.3). In particular, we have $\beta_{Y}^{\varepsilon}(0)=\beta_{\Gamma_{Y}}^{\varepsilon}(0)=0$. 
(A4) The functions $\pi, \pi_{\Gamma}: \mathbb{R} \rightarrow \mathbb{R}$ are Lipschitz continuous.

(A5) The function $g: \overline{D(\beta)} \rightarrow[0,+\infty)$ belongs to $C^{2}$, is bounded and concave, and $g^{\prime}$ is bounded and Lipschitz continuous.

(A6) $\widehat{\beta}\left(\rho_{0}\right) \in L^{1}(\Omega), \quad \widehat{\beta}_{\Gamma}\left(\rho_{0_{\Gamma}}\right) \in L^{1}(\Gamma), \quad \beta^{\circ}\left(\rho_{0}\right) \in H, \quad \beta_{\Gamma}^{\circ}\left(\rho_{0_{\Gamma}}\right) \in H_{\Gamma}$.

Finally, we need a compatibility condition, which essentially means that the graph in the bulk is dominated by the graph on the boundary:

(A7) $D\left(\beta_{\Gamma}\right) \subset D(\beta)$, and there are constants $\eta>0$ and $C_{\Gamma} \geq 0$ such that

$$
\left|\beta^{\circ}(r)\right| \leq \eta\left|\beta_{\Gamma}^{\circ}(r)\right|+C_{\Gamma} \quad \forall r \in D\left(\beta_{\Gamma}\right) .
$$

We now state the main results of this paper. Concerning well-posedness, we have the following result.

THEOREM 2.1: Suppose that the assumptions (A1)-(A7) are fulfilled. Then the system (1.1)-(1.7) admits a unique solution $\left(\mu, \rho, \rho_{\Gamma}, \xi, \xi_{\Gamma}\right)$ such that $\mu \geq 0$ almost everywhere in $Q$ and

$$
\begin{aligned}
& \mu \in W^{1, p}(0, T ; H) \cap C^{0}([0, T] ; V) \cap L^{p}(0, T ; W) \cap L^{\infty}(Q) \quad \text { for every } p \in[1,+\infty), \\
& \rho \in W^{1, \infty}(0, T ; H) \cap H^{1}(0, T ; V) \cap L^{\infty}\left(0, T ; H^{2}(\Omega)\right) \\
& \rho_{\Gamma} \in W^{1, \infty}\left(0, T, H_{\Gamma}\right) \cap H^{1}\left(0, T, V_{\Gamma}\right) \cap L^{\infty}\left(0, T ; H^{2}(\Gamma)\right) \\
& \xi \in L^{\infty}(0, T ; H), \quad \xi_{\Gamma} \in L^{\infty}\left(0, T ; H_{\Gamma}\right) .
\end{aligned}
$$

REMARK 2.2: Observe that it follows from standard embedding results (see, e.g., [45, Sect. 8, Cor. 4]) that $\rho \in C^{0}\left([0, T] ; H^{s}(\Omega)\right)$ for $0<s<2$. In particular, $\rho \in C^{0}(\bar{Q})$, which entails that $\rho_{\Gamma}=\rho_{\mid \Sigma} \in C^{0}(\bar{\Sigma})$. Notice also that it follows from (2.5) and (2.6) that both $|\nabla \mu|$ and $|\nabla \rho|$ belong to $L^{4}\left(0, T ; L^{6}(\Omega)\right)$.

Under somewhat stronger assumptions, we can prove that the solution enjoys a better regularity. More precisely, we have the following result.

Theorem 2.3: $\quad$ Suppose that (A1)-(A7) hold true, and let

$$
u_{\Gamma} \in L^{\infty}(\Sigma), \quad \beta^{\circ}\left(\rho_{0}\right) \in L^{\infty}(\Omega) \quad \text { and } \quad \beta^{\circ}\left(\rho_{0_{\Gamma}}\right) \in L^{\infty}(\Gamma) .
$$

Then, the unique solution $\left(\mu, \rho, \rho_{\Gamma}, \xi, \xi_{\Gamma}\right)$ to (1.1)-(1.7) established in Theorem 2.1 also satisfies $\xi \in L^{\infty}(Q)$.

The above theorems hold for every pair of potentials $\widehat{\beta}$ and $\widehat{\beta}_{\Gamma}$ satisfying assumptions (A3) and (A7). In our last result, we reinforce the compatibility condition a little and consider a more restricted class of potentials which does not include potentials of obstacle type, while potentials of logarithmic type are still admitted. Namely, we assume that

$$
-\infty \leq r_{-}<r_{+} \leq+\infty \quad \text { and } \quad D(\beta)=D\left(\beta_{\Gamma}\right)=\left(r_{-}, r_{+}\right)
$$


and prove a uniform separation property. We observe that such an assumption allows multi-valued subdifferentials. A stability estimate holds if in addition the potentials are smooth.

TheOREm 2.4: Assume that (A1)-(A8) and (2.9) are fulfilled. Then, the unique solution $\left(\mu, \rho, \rho_{\Gamma}, \xi, \xi_{\Gamma}\right)$ to (1.1)-(1.7) established in Theorem 2.1 satisfies the uniform separation property

$$
r_{*} \leq \rho(x, t) \leq r^{*}, \quad \text { for every }(x, t) \in \bar{Q},
$$

with constants $r_{*}, r^{*} \in\left(r_{-}, r_{+}\right)$that depend only on the data of the system, and we also have $\xi_{\Gamma} \in L^{\infty}(\Sigma)$. Moreover, assume that $\widehat{\beta}$ and $\widehat{\beta}_{\Gamma}$ are functions of class $C^{2}$ in $\left(r_{-}, r_{+}\right)$and let $u_{i_{\Gamma}} \in H^{1}\left(0, T ; H_{\Gamma}\right) \cap L^{\infty}(\Sigma)$ be given. Then, the corresponding solutions $\left(\mu_{i}, \rho_{i}, \rho_{i_{\Gamma}}, \xi_{i}, \xi_{i_{\Gamma}}\right)$ satisfy the stability estimate

$$
\begin{aligned}
& \left\|\mu_{1}-\mu_{2}\right\|_{L^{\infty}(0, t ; H) \cap L^{2}([0, t] ; V)}+\left\|\rho_{1}-\rho_{2}\right\|_{H^{1}(0, t ; H) \cap C^{0}([0, t] ; V) \cap L^{2}\left(0, t ; H^{2}(\Omega)\right)} \\
& \quad+\left\|\rho_{1_{\Gamma}}-\rho_{2_{\Gamma}}\right\|_{H^{1}\left(0, t ; H_{\Gamma}\right) \cap C^{0}\left([0, t] ; V_{\Gamma}\right) \cap L^{2}\left(0, t ; H^{2}(\Gamma)\right)} \\
& \leq \widehat{C}\left\|u_{1_{\Gamma}}-u_{2_{\Gamma}}\right\|_{L^{2}\left(0, t ; H_{\Gamma}\right)}, \quad \forall t \in(0, T]
\end{aligned}
$$

with a finite constant $\widehat{C}>0$ that depends only on the data of the system.

REMARK 2.5: If $\left(r_{-}, r_{+}\right)$is bounded (but a similar remark holds in the case of a half line), then both $\beta$ and $\beta_{\Gamma}$ become singular at $r_{ \pm}$due to maximal monotonicity. However, such a singularity never becomes active thanks to (2.10).

\section{$3 \quad$ Existence and uniqueness}

In this section, we prove the relevant well-posedness result.

Proof of Theorem 2.1: We employ an approximation scheme based on Yosida approximation and a time delay in the right-hand side of (1.3). To this end, let $\beta^{\varepsilon}:=\beta_{Y}^{\varepsilon}$ and $\beta_{\Gamma}^{\varepsilon}:=\beta_{\Gamma_{Y}}^{\varepsilon \eta}$ denote the Yosida approximations of $\beta$ and $\beta_{\Gamma}$ at the levels $\varepsilon>0$ and $\varepsilon \eta>0$, respectively, where $\eta>0$ is the constant introduced in (2.4). Then $\beta^{\varepsilon}, \beta_{\Gamma}^{\varepsilon}$ satisfy $\beta^{\varepsilon}(0)=\beta_{\Gamma}^{\varepsilon}(0)=0$ and the conditions (2.1)-(2.3) correspondingly. Moreover, we infer from [5, Lemma 4.4] that

$$
\left|\beta^{\varepsilon}(r)\right| \leq \eta\left|\beta_{\Gamma}^{\varepsilon}(r)\right|+C_{\Gamma} \quad \forall r \in \mathbb{R} .
$$

Since, by virtue of their monotonicity, the functions $\beta^{\varepsilon}$ and $\beta_{\Gamma}^{\varepsilon}$ have equal sign, it follows from (3.1) and Young's inequality for all $r \in \mathbb{R}$ the estimate

$$
\beta^{\varepsilon}(r) \beta_{\Gamma}^{\varepsilon}(r)=\left|\beta^{\varepsilon}(r)\right|\left|\beta_{\Gamma}^{\varepsilon}(r)\right| \geq \frac{1}{\eta}\left|\beta^{\varepsilon}(r)\right|^{2}-\frac{C_{\Gamma}}{\eta}\left|\beta^{\varepsilon}(r)\right| \geq \frac{1}{2 \eta}\left|\beta^{\varepsilon}(r)\right|^{2}-\frac{C_{\Gamma}^{2}}{2 \eta} .
$$

We also temporarily extend the function $g$ to the whole real line $\mathbb{R}$, still terming the extended function $g$, in such a way that

$$
\begin{aligned}
& g \in C^{1}(\mathbb{R}), g \text { and } g^{\prime} \text { are bounded and Lipschitz continuous on } \mathbb{R} \text {, and } \\
& g(r) \geq-1 / 3 \quad \text { (i. e., } 1+2 g(r) \geq 1 / 3>0) \quad \forall r \in \mathbb{R} .
\end{aligned}
$$


To introduce the time delay, we define for every $\tau \in(0, T)$ the translation operator $\mathcal{T}_{\tau}: C^{0}([0, T] ; H) \rightarrow C^{0}([0, T] ; H)$ by setting, for all $v \in C^{0}([0, T] ; H)$,

$$
\mathcal{T}_{\tau}(v)(t):=v(t-\tau) \quad \text { if } t>\tau \quad \text { and } \mathcal{T}_{\tau}(v)(t):=v(0) \quad \text { if } t \leq \tau
$$

Notice that for every $v \in H^{1}(0, T ; H)$ it holds that

$$
\begin{gathered}
\left\|\mathcal{T}_{\tau}(v)\right\|_{L^{2}\left(Q_{t}\right)}^{2} \leq\left\{\begin{array}{l}
\|v\|_{L^{2}\left(Q_{t}\right)}^{2}+\tau\|v(0)\|_{H}^{2} \quad \text { for all } t \in[\tau, T], \\
t\|v(0)\|_{H}^{2}
\end{array} \text { for all } t \in[0, \tau],\right. \\
\left\|\partial_{t} \mathcal{T}_{\tau}(v)\right\|_{L^{2}\left(Q_{t}\right)}^{2} \leq\left\|\partial_{t} v\right\|_{L^{2}\left(Q_{t}\right)}^{2} \quad \text { for a. e. } t \in(0, T),
\end{gathered}
$$

while for every $v \in C^{0}([0, T] ; V)$ we have

$$
\left\|\nabla \mathcal{T}_{\tau}(v)\right\|_{L^{2}\left(Q_{t}\right)}^{2} \leq\left\{\begin{array}{l}
\|\nabla v\|_{L^{2}\left(Q_{t}\right)}^{2}+\tau\|\nabla v(0)\|_{H}^{2} \quad \text { for all } t \in[\tau, T] \\
t\|\nabla v(0)\|_{H}^{2}
\end{array} \text { for all } t \in[0, \tau] .\right.
$$

We then consider the problem (which in the following will be termed $\left(P_{\tau}^{\varepsilon}\right)$ ) of finding a triple $\left(\mu_{\tau}^{\varepsilon}, \rho_{\tau}^{\varepsilon}, \rho_{\tau_{\Gamma}}^{\varepsilon}\right)$ with

$$
\begin{aligned}
& \mu_{\tau}^{\varepsilon} \in H^{1}(0, T ; H) \cap C^{0}([0, T] ; V) \cap L^{2}(0, T ; W) \cap L^{\infty}(Q), \quad \mu_{\tau}^{\varepsilon} \geq 0 \quad \text { a. e. in } Q, \\
& \rho_{\tau}^{\varepsilon} \in W^{1, \infty}(0, T ; H) \cap H^{1}(0, T ; V) \cap L^{2}\left(0, T ; H^{2}(\Omega)\right), \\
& \left.\rho_{\tau_{\Gamma}}^{\varepsilon} \in W^{1, \infty}\left(0, T ; H_{\Gamma}\right) \cap H^{1}\left(0, T ; V_{\Gamma}\right)\right) \cap L^{2}\left(0, T ; H^{2}(\Gamma)\right),
\end{aligned}
$$

which solves the initial-boundary value problem

$$
\begin{aligned}
& \left(1+2 g\left(\rho_{\tau}^{\varepsilon}\right)\right) \partial_{t} \mu_{\tau}^{\varepsilon}+g^{\prime}\left(\rho_{\tau}^{\varepsilon}\right) \partial_{t} \rho_{\tau}^{\varepsilon} \mu_{\tau}^{\varepsilon}-\Delta \mu_{\tau}^{\varepsilon}=0 \quad \text { a. e. in } Q, \\
& \partial_{\mathbf{n}} \mu_{\tau}^{\varepsilon}=0 \quad \text { a. e. on } \Sigma \\
& \partial_{t} \rho_{\tau}^{\varepsilon}-\Delta \rho_{\tau}^{\varepsilon}+\beta^{\varepsilon}\left(\rho_{\tau}^{\varepsilon}\right)+\pi\left(\rho_{\tau}^{\varepsilon}\right)=\mathcal{T}_{\tau}\left(\mu_{\tau}^{\varepsilon}\right) g^{\prime}\left(\rho_{\tau}^{\varepsilon}\right) \quad \text { a. e. in } Q, \\
& \partial_{\mathbf{n}} \rho_{\tau}^{\varepsilon}+\partial_{t} \rho_{\tau_{\Gamma}}^{\varepsilon}+\beta_{\Gamma}^{\varepsilon}\left(\rho_{\tau_{\Gamma}}^{\varepsilon}\right)+\pi_{\Gamma}\left(\rho_{\tau_{\Gamma}}^{\varepsilon}\right)-\Delta_{\Gamma} \rho_{\tau_{\Gamma}}^{\varepsilon}=u_{\Gamma}, \quad \rho_{\tau_{\Gamma}}^{\varepsilon}=\rho_{\tau_{\mid \Sigma}}^{\varepsilon}, \quad \text { a. e. on } \Sigma, \\
& \mu_{\tau}^{\varepsilon}(0)=\mu_{0} \quad \text { and } \quad \rho_{\tau}^{\varepsilon}(0)=\rho_{0} \quad \text { a. e. in } \Omega, \quad \rho_{\tau_{\Gamma}}^{\varepsilon}(0)=\rho_{0_{\mid \Gamma}} \quad \text { a.e. on } \Gamma \text {. }
\end{aligned}
$$

For convenience, we allow $\tau$ to attain just discrete values, namely, $\tau=\tau_{N}:=\frac{T}{N}$, where $N$ is any positive integer. We now proceed in four steps: at first, we show that the problem $\left(P_{\tau}^{\varepsilon}\right)$ is well posed for every $\tau=\tau_{N}, N \in \mathbb{N}$, then we prove a number of a priori estimates, followed by the limit processes as $N \rightarrow \infty$ and then as $\varepsilon \searrow 0$. The limit processes use standard compactness and monotonicity arguments. As a notational convention, in the remainder of this proof we will denote by $K_{i}, i \in \mathbb{N}$, positive constants that may depend on the data of the system (1.1)-(1.7) but neither on $\tau>0$ nor on $\varepsilon>0$.

STEP 1: We claim that, for every $\tau_{N}=\frac{T}{N}, N \in \mathbb{N}$, and every $\varepsilon>0$, the problem (3.9)-(3.13) admits a unique solution triple $\left(\mu_{\tau_{N}}^{\varepsilon}, \rho_{\tau_{N}}^{\varepsilon}, \rho_{\tau_{N_{\Gamma}}}^{\varepsilon}\right)$ satisfying (3.8).

To prove this claim, we set $t_{n}:=n \tau_{N}$ for $n=0, \ldots, N$, and observe that the problem of solving (3.9)-(3.13) becomes equivalent to a finite sequence of $N$ problems that can be solved inductively step by step. However, instead of considering the natural time intervals $\left[t_{n-1}, t_{n}\right], n=1, \ldots, N$ and glueing the solutions together, we solve $N$ problems on the 
time intervals $I_{n}=\left[0, t_{n}\right], n=1, \ldots, N$, by constructing the solution directly on the whole of $I_{n}$ at each step. These problems read as follows:

$$
\begin{aligned}
& \left(1+2 g\left(\rho_{n}\right)\right) \partial_{t} \mu_{n}+g^{\prime}\left(\rho_{n}\right) \partial_{t} \rho_{n} \mu_{n}-\Delta \mu_{n}=0 \quad \text { and } \quad \mu_{n} \geq 0 \quad \text { a.e. in } \Omega \times I_{n} \text {, } \\
& \partial_{\mathbf{n}} \mu_{n}=0 \text { a.e. on } \Gamma \times I_{n} \text { and } \mu_{n}(0)=\mu_{0} \text { a.e. in } \Omega \text {, } \\
& \partial_{t} \rho_{n}-\Delta \rho_{n}+\beta^{\varepsilon}\left(\rho_{n}\right)+\pi\left(\rho_{n}\right)=\mathcal{T}_{\tau_{N}}\left(\mu_{n-1}\right) g^{\prime}\left(\rho_{n}\right) \text { a. e. in } \Omega \times I_{n} \text {, } \\
& \partial_{\mathbf{n}} \rho_{n}+\partial_{t} \rho_{n_{\Gamma}}+\beta_{\Gamma}^{\varepsilon}\left(\rho_{n_{\Gamma}}\right)+\pi_{\Gamma}\left(\rho_{n_{\Gamma}}\right)-\Delta_{\Gamma} \rho_{n_{\Gamma}}=u_{\Gamma}, \\
& \rho_{n_{\Gamma}}=\rho_{n_{\mid \Gamma \times I_{n}}}, \quad \text { a.e. on } \Gamma \times I_{n}, \\
& \rho_{n}(0)=\rho_{0} \quad \text { a.e. in } \Omega, \quad \rho_{n_{\Gamma}}(0)=\rho_{0_{\mid \Gamma}} \quad \text { a. e. on } \Gamma \text {. }
\end{aligned}
$$

Their (unique) solutions $\left(\mu_{n}, \rho_{n}, \rho_{n_{\Gamma}}\right)$ are required to satisfy the regularity properties inductively obtained by taking $t_{n}$ in place of $T$ in (3.8). The operator $\mathcal{T}_{\tau_{N}}$ appearing on the right-hand side of (3.16) acts on functions that are not defined in the whole of $(0, T)$; however, its meaning is still given by (3.4) if $n>1$, while for $n=1$ we simply set $\mathcal{T}_{\tau_{N}}\left(\mu_{n-1}\right)=\mu_{0}$.

Observe that the unique solvability of (3.14)-(3.18) for every $n \in\{1, \ldots, N\}$ would imply that

$$
\rho_{N_{\mid \Omega \times I_{N-1}}}=\rho_{N-1}, \quad \mu_{N_{\mid \Omega \times I_{N-1}}}=\mu_{N-1},
$$

which would entail that

$$
\begin{array}{r}
\mathcal{T}_{\tau_{N}}\left(\mu_{N-1}\right)(x, t)=\mu_{N-1}\left(x, t-\tau_{N}\right)=\mu_{N}\left(x, t-\tau_{N}\right)=\mathcal{T}_{\tau_{N}}\left(\mu_{N}\right)(x, t) \\
\text { for a. e. }(x, t) \in \Omega \times I_{N-1} .
\end{array}
$$

It then would follow that $\left(\mu_{N}, \rho_{N}, \rho_{N_{\Gamma}}\right)$ is the unique solution to the system (3.9)-(3.13) for $\tau=\tau_{N}$. Consequently, it suffices to show that the system (3.14)-(3.18) enjoys for every $n \in\{1, \ldots, N\}$ a unique solution having the required regularity. To this end, we argue by induction. Since the proof for $n=1$ is similar to that used in the induction step $n-1 \longrightarrow n$ (recall that we have set $\mathcal{T}_{\tau_{N}}\left(\mu_{0}\right)=\mu_{0}$ on $\Omega \times\left(0, t_{1}\right)$ ), we may confine ourselves to just perform the latter.

So let $1<n \leq N$, and assume that, for $1 \leq k \leq n-1$, unique solutions $\left(\mu_{k}, \rho_{k}, \rho_{k_{\Gamma}}\right)$ to the system (3.14)-(3.18) have already been constructed that satisfy for $1 \leq k \leq n-1$ the conditions

$$
\begin{aligned}
& \mu_{k} \in H^{1}\left(I_{k} ; H\right) \cap C^{0}\left(\bar{I}_{k} ; V\right) \cap L^{2}\left(I_{k} ; W\right) \cap L^{\infty}\left(\Omega \times I_{k}\right), \quad \mu_{k} \geq 0 \quad \text { a. e. in } \Omega \times I_{k}, \\
& \rho_{k} \in W^{1, \infty}\left(I_{k} ; H\right) \cap H^{1}\left(I_{k} ; V\right) \cap L^{2}\left(I_{k} ; H^{2}(\Omega)\right), \\
& \left.\rho_{k_{\Gamma}} \in W^{1, \infty}\left(I_{k} ; H_{\Gamma}\right) \cap H^{1}\left(I_{k} ; V_{\Gamma}\right)\right) \cap L^{2}\left(I_{k} ; H^{2}(\Gamma)\right) .
\end{aligned}
$$

Now observe that the system (3.16)-(3.18) is nothing but the strong formulation of the variational problem [5, Eqs. (4.5)-(4.8)] (if one puts, in the notation used there, $\left.f_{\Gamma}:=u_{\Gamma}, f=0\right)$, with the only exception that the expression $\pi\left(\rho_{n}^{\varepsilon}\right)$ occurring there is in our case replaced by $\pi\left(\rho_{n}^{\varepsilon}\right)-\mathcal{T}_{\tau_{N}}\left(\mu_{n-1}\right) g^{\prime}\left(\rho_{n}^{\varepsilon}\right)$. Now notice that the data of the system satisfy all of the conditions imposed in [5]. In view of (3.3), and since $\mathcal{T}_{\tau_{N}}\left(\mu_{n-1}\right)$ obviously belongs to $L^{\infty}\left(\Omega \times I_{n}\right)$, we can therefore adopt the arguments employed in [5, Sect. 4] (see, in particular, [5, Prop. 4.1 and Rem. 4.5]) to conclude that (3.16)-(3.18) enjoys a 
unique solution $\left(\rho_{n}, \rho_{n_{\Gamma}}\right)$ such that

$$
\begin{aligned}
& \rho_{n} \in H^{1}\left(I_{n} ; H\right) \cap C^{0}\left(\bar{I}_{n} ; V\right) \cap L^{2}\left(I_{n} ; H^{2}(\Omega)\right), \\
& \rho_{n_{\Gamma}} \in H^{1}\left(I_{n} ; H_{\Gamma}\right) \cap C^{0}\left(\bar{I}_{n} ; V_{\Gamma}\right) \cap L^{2}\left(I_{n} ; H^{2}(\Gamma)\right) \\
& \beta^{\varepsilon}\left(\rho_{n}\right) \in L^{2}\left(I_{n} ; H\right), \quad \beta_{\Gamma}^{\varepsilon}\left(\rho_{n_{\Gamma}}\right) \in L^{2}\left(I_{n} ; H_{\Gamma}\right) .
\end{aligned}
$$

In order to recover the full regularity required in (3.8), we still need to show that

$$
\partial_{t} \rho_{n} \in L^{\infty}\left(I_{n} ; H\right) \cap L^{2}\left(I_{n} ; V\right), \quad \partial_{t} \rho_{n_{\Gamma}} \in L^{\infty}\left(I_{n} ; H_{\Gamma}\right) \cap L^{2}\left(I_{n} ; V_{\Gamma}\right) .
$$

To establish (3.23), we proceed formally, noting that the following arguments can be made rigorous by applying, e.g., finite differences in time. We differentiate (3.16) (formally) with respect to time, obtaining the identity

$$
\begin{aligned}
& \partial_{t t}^{2} \rho_{n}-\Delta \partial_{t} \rho_{n}+\left(\beta^{\varepsilon}\right)^{\prime}\left(\rho_{n}\right) \partial_{t} \rho_{n} \\
& =\left(-\pi^{\prime}\left(\rho_{n}\right)+\mathcal{T}_{\tau_{n}}\left(\mu_{n-1}\right) g^{\prime \prime}\left(\rho_{n}\right)\right) \partial_{t} \rho_{n}+\partial_{t} \mathcal{T}_{\tau_{n}}\left(\mu_{n-1}\right) g^{\prime}\left(\rho_{n}\right) .
\end{aligned}
$$

Multiplication by $\partial_{t} \rho_{n}$, integration over $Q_{t}$, where $t \in(0, T]$, and (formal) integration by parts, using (3.17), yields the identity

$$
\begin{aligned}
& \frac{1}{2}\left(\left\|\partial_{t} \rho_{n}(t)\right\|_{H}^{2}+\left\|\partial_{t} \rho_{n_{\Gamma}}(t)\right\|_{H_{\Gamma}}^{2}\right)+\int_{0}^{t} \int_{\Omega}\left|\nabla \partial_{t} \rho_{n}\right|^{2} \mathrm{~d} x \mathrm{~d} s+\int_{0}^{t} \int_{\Gamma}\left|\nabla_{\Gamma} \partial_{t} \rho_{n_{\Gamma}}\right|^{2} \mathrm{~d} \Gamma \mathrm{d} s \\
& +\int_{0}^{t} \int_{\Omega}\left(\beta^{\varepsilon}\right)^{\prime}\left(\rho_{n}\right)\left|\partial_{t} \rho_{n}\right|^{2} \mathrm{~d} x \mathrm{~d} s+\int_{0}^{t} \int_{\Gamma}\left(\beta_{\Gamma}^{\varepsilon}\right)^{\prime}\left(\rho_{n_{\Gamma}}\right)\left|\partial_{t} \rho_{n_{\Gamma}}\right|^{2} \mathrm{~d} \Gamma \mathrm{d} s \\
& =\frac{1}{2}\left(\left\|\partial_{t} \rho_{n}(0)\right\|_{H}^{2}+\left\|\partial_{t} \rho_{n_{\Gamma}}(0)\right\|_{H_{\Gamma}}^{2}\right)+\int_{0}^{t} \int_{\Omega}\left(-\pi^{\prime}\left(\rho_{n}\right)+\mathcal{T}_{\tau_{n}}\left(\mu_{n-1}\right) g^{\prime \prime}\left(\rho_{n}\right)\right)\left|\partial_{t} \rho_{n}\right|^{2} \mathrm{~d} x \mathrm{~d} t \\
& \quad+\int_{0}^{t} \int_{\Omega} \partial_{t} \mathcal{T}_{\tau_{n}}\left(\mu_{n-1}\right) g^{\prime}\left(\rho_{n}\right) \partial_{t} \rho_{n} \mathrm{~d} x \mathrm{~d} s+\int_{0}^{t} \int_{\Gamma}\left(\partial_{t} u_{\Gamma}-\pi_{\Gamma}^{\prime}\left(\rho_{n_{\Gamma}}\right) \partial_{t} \rho_{n_{\Gamma}}\right) \partial_{t} \rho_{n_{\Gamma}} \mathrm{d} \Gamma \mathrm{d} s . \quad(3.2)
\end{aligned}
$$

By the monotonicity of $\beta^{\varepsilon}$ and $\beta_{\Gamma}^{\varepsilon}$, all of the terms on the left-hand side are nonnegative, and, invoking (A2), (3.6), the boundedness of $\pi^{\prime}, \pi_{\Gamma}^{\prime}, g^{\prime}, g^{\prime \prime}, \mu_{n-1}$, and Young's inequality, we readily conclude from (3.20) and the first (3.19) with $k=n-1$ that the three integrals on the right-hand side are finite.

Moreover, by (A1), (A4), (A5) and (A6), we have $\mu_{0} g^{\prime}\left(\rho_{0}\right)+\Delta \rho_{0}-\pi\left(\rho_{0}\right) \in H$, and it follows from (2.1) that, for every $\varepsilon>0$,

$$
\left\|\beta^{\varepsilon}\left(\rho_{0}\right)\right\|_{H} \leq\left\|\beta^{\circ}(\rho)\right\|_{H} \quad \text { and } \quad\left\|\beta_{\Gamma}^{\varepsilon}\left(\rho_{0_{\Gamma}}\right)\right\|_{H_{\Gamma}} \leq\left\|\beta_{\Gamma}^{\circ}\left(\rho_{0_{\Gamma}}\right)\right\|_{H_{\Gamma}} .
$$

We thus can infer from (A6) that

$$
\left\|\partial_{t} \rho_{n}(0)\right\|_{H}=\left\|\mu_{0} g^{\prime}\left(\rho_{0}\right)+\Delta \rho_{0}-\pi\left(\rho_{0}\right)-\beta^{\varepsilon}\left(\rho_{0}\right)\right\|_{H} \leq K_{1} .
$$

Likewise, using (A1), (A2), (A4), (A6), and (2.1), we conclude that

$$
\left\|\partial_{t} \rho_{n_{\Gamma}}(0)\right\|_{H_{\Gamma}}=\left\|u_{\Gamma}(0)-\partial_{\mathbf{n}} \rho_{0}+\Delta_{\Gamma} \rho_{0_{\Gamma}}-\pi_{\Gamma}\left(\rho_{0_{\Gamma}}\right)-\beta_{\Gamma}^{\varepsilon}\left(\rho_{0_{\Gamma}}\right)\right\|_{H_{\Gamma}} \leq K_{2} .
$$

In conclusion, the expression on the right-hand side of (3.24) is finite, which proves the additional regularity (3.23). 
Now that the existence of a unique solution $\rho_{n}$ to the system (3.16)-(3.18) with the required regularity is established, we substitute it in (3.14) and study the resulting initial-boundary value problem (3.14)-(3.15). Recalling the continuity of the embedding

$$
\left(L^{\infty}(0, t ; H) \cap L^{2}(0, t ; V)\right) \subset\left(L^{10 / 3}\left(Q_{t}\right) \cap L^{7 / 3}\left(0, t ; L^{14 / 3}(\Omega)\right)\right) \quad \forall t \in(0, T],
$$

we have that

$$
\partial_{t} \rho_{n} \in L^{10 / 3}\left(Q_{t_{n}}\right) \cap L^{7 / 3}\left(0, t_{n} ; L^{14 / 3}(\Omega)\right) .
$$

We now recall the form (3.3) of the extension of $g$ to the whole real line. Using this, we may argue as in [25, pp. 7953-7956] to conclude that, with $\rho_{n}$ fixed, the problem (3.14)-(3.15) has a unique nonnegative solution $\mu_{n} \in H^{1}\left(I_{n} ; H\right) \cap L^{\infty}\left(I_{n} ; V\right) \cap L^{2}\left(I_{n} ; W^{2,3 / 2}(\Omega)\right)$. But since $\partial_{t} \rho_{n} \in L^{2}\left(I_{n} ; V\right)$, it follows from the continuity of the embedding $V \subset L^{6}(\Omega)$ that $g^{\prime}\left(\rho_{n}\right) \partial_{t} \rho_{n} \mu_{n} \in L^{2}\left(I_{n} ; H\right)$, and comparison in (3.14) shows that $\Delta \mu_{n} \in L^{2}\left(I_{n} ; H\right)$, whence, by standard elliptic estimates, also $\mu_{n} \in L^{2}\left(I_{n} ; W\right)$. The continuity of the embedding $\left(H^{1}\left(I_{n} ; H\right) \cap L^{2}\left(I_{n} ; H^{2}(\Omega)\right)\right) \subset C^{0}\left(\bar{I}_{n} ; V\right)$ yields that then also $\mu_{n} \in C^{0}\left(\bar{I}_{n} ; V\right)$.

Finally, we have $\partial_{t} \rho_{n} \in L^{7 / 3}\left(I_{n} ; L^{14 / 3}(\Omega)\right)$, by (3.29). Therefore, we may repeat the argument from the proof of [14, Thm. 2.3], which was based on this regularity, to conclude that $\mu_{n} \in L^{\infty}\left(\Omega \times I_{n}\right)$. Here, we remark that the quoted proof was performed only for the special case $g(r)=r$; however, the argument extends with only minor modifications to the present situation (see also the analogous [19, Thm. 3.7]). With this, the above claim is completely proved.

STEP 2: $\quad$ Let $\varepsilon>0$ and $N \in \mathbb{N}$ be arbitrary but fixed, and let $\left(\mu_{\tau_{N}}^{\varepsilon}, \rho_{\tau_{N}}^{\varepsilon}, \rho_{\tau_{N_{\Gamma}}}^{\varepsilon}\right)$ be the unique solution to $\left(P_{\tau_{N}}^{\varepsilon}\right)$ having the regularity properties (3.8), which was constructed in Step 1. We are now going to show a number of a priori estimates for these solutions which are uniform with respect to $N$ and $\varepsilon>0$. In performing these estimates, for the sake of a better readability we will omit both the subscript $\tau_{N}$ and the superscript $\varepsilon$, writing them only at the end of each estimate. We also recall that $K_{i}, i \in \mathbb{N}$, denote positive constants which are independent of both $\varepsilon$ and $N$. We remark that the following chain of estimates combines ideas from [19] and [5], where in [19] a more general version of Eq. (1.1) was investigated. Since, however, in [19] the simpler case of a zero Neumann boundary condition for $\rho$ was assumed in place of the dynamic boundary condition (1.5), we have chosen to include these estimates for the reader's convenience.

\section{FIRST ESTIMATE:}

First observe that $\partial_{t}\left(\left(\frac{1}{2}+g(\rho)\right) \mu^{2}\right)=(1+2 g(\rho)) \mu \partial_{t} \mu+g^{\prime}(\rho) \partial_{t} \rho \mu^{2}$. Hence, multiplying (3.9) by $\mu$, and integrating over $Q_{t}$, where $t \in(0, T]$, we find the identity

$$
\int_{\Omega}\left(\frac{1}{2}+g(\rho(t))\right) \mu^{2}(t) \mathrm{d} x+\int_{0}^{t} \int_{\Omega}|\nabla \mu|^{2} \mathrm{~d} x \mathrm{~d} s=\int_{\Omega}\left(\frac{1}{2}+g\left(\rho_{0}\right)\right) \mu_{0}^{2}(t) \mathrm{d} x,
$$

whence, using (A1), we easily conclude that

$$
\left\|\mu_{\tau_{N}}^{\varepsilon}\right\|_{L^{\infty}(0, T ; H) \cap L^{2}(0, T ; V)} \leq K_{3},
$$

which entails that also

$$
\left\|\mathcal{T}_{\tau_{N}}\left(\mu_{\tau_{N}}^{\varepsilon}\right)\right\|_{L^{\infty}(0, T ; H) \cap L^{2}(0, T ; V)} \leq K_{4}
$$




\section{SECOND ESTIMATE:}

Next, we add $\rho$ on both sides of (3.11), multiply by $\partial_{t} \rho$, and integrate over $Q_{t}$, where $t \in(0, T]$. Adding $\rho_{\Gamma}$ on both sides of $(3.12)$, we then obtain the identity

$$
\begin{aligned}
& \int_{0}^{t} \int_{\Omega}\left|\partial_{t} \rho\right|^{2} \mathrm{~d} x \mathrm{~d} s+\frac{1}{2}\|\rho(t)\|_{V}^{2}+\int_{\Omega} \widehat{\beta}^{\varepsilon}(\rho(t)) \mathrm{d} x \\
& +\int_{0}^{t} \int_{\Gamma}\left|\partial_{t} \rho_{\Gamma}\right|^{2} \mathrm{~d} \Gamma \mathrm{d} s+\frac{1}{2}\left\|\rho_{\Gamma}(t)\right\|_{V_{\Gamma}}^{2}+\int_{\Gamma} \widehat{\beta}_{\Gamma}^{\varepsilon}\left(\rho_{\Gamma}(t)\right) \mathrm{d} \Gamma \\
& =\frac{1}{2}\left\|\rho_{0}\right\|_{V}^{2}+\int_{\Omega} \widehat{\beta}^{\varepsilon}\left(\rho_{0}\right) \mathrm{d} x+\frac{1}{2}\left\|\rho_{0_{\Gamma}}\right\|_{V_{\Gamma}}^{2}+\int_{\Gamma} \widehat{\beta}_{\Gamma}^{\varepsilon}\left(\rho_{0_{\Gamma}}\right) \mathrm{d} \Gamma \\
& +\int_{0}^{t} \int_{\Omega} \partial_{t} \rho\left(\mathcal{T}_{\tau_{N}}(\mu) g^{\prime}(\rho)-\pi(\rho)+\rho\right) \mathrm{d} x \mathrm{~d} s+\int_{0}^{t} \int_{\Gamma} \partial_{t} \rho_{\Gamma}\left(u_{\Gamma}-\pi_{\Gamma}\left(\rho_{\Gamma}\right)+\rho_{\Gamma}\right) \mathrm{d} \Gamma \mathrm{d} s .
\end{aligned}
$$

From (2.3), we obtain that all of the expressions on the left-hand side are nonnegative, and it follows from (A1), (2.3), and (A6), that the first four summands on the right-hand side are bounded by a constant that neither depends on $N \in \mathbb{N}$ nor on $\varepsilon>0$. The last two integrals on the right-hand side, which we denote by $I_{1}$ and $I_{2}$, respectively, can be estimated as follows: we employ (3.3), (3.31), (A4), and Young's inequality, to obtain that

$$
I_{1} \leq \frac{1}{2} \int_{0}^{t} \int_{\Omega}\left|\partial_{t} \rho\right|^{2} \mathrm{~d} x \mathrm{~d} s+K_{5}\left(1+\int_{0}^{t} \int_{\Omega}|\rho|^{2} \mathrm{~d} x \mathrm{~d} s\right),
$$

and invoke (A2), (A4), and Young's inequality, to see that

$$
I_{2} \leq \frac{1}{2} \int_{0}^{t} \int_{\Gamma}\left|\partial_{t} \rho_{\Gamma}\right|^{2} \mathrm{~d} \Gamma \mathrm{d} s+K_{6}\left(1+\int_{0}^{t} \int_{\Gamma}\left|\rho_{\Gamma}\right|^{2} \mathrm{~d} \Gamma \mathrm{d} s\right) .
$$

We thus can infer from Gronwall's lemma that

$$
\left\|\rho_{\tau_{N}}^{\varepsilon}\right\|_{H^{1}(0, T ; H) \cap L^{\infty}(0, T ; V)}+\| \rho_{\tau_{N_{\Gamma}}^{\varepsilon}}^{\|_{H^{1}\left(0, T ; H_{\Gamma}\right) \cap L^{\infty}\left(0, T ; V_{\Gamma}\right)}} \leq K_{7} .
$$

\section{THIRD ESTIMATE:}

Next, we multiply (3.11) by $\beta^{\varepsilon}(\rho)$ and integrate over $Q_{t}$, where $0<t \leq T$. Then, we integrate by parts and use (3.12)-(3.13) to obtain the identity

$$
\begin{aligned}
& \int_{\Omega} \widehat{\beta}^{\varepsilon}(\rho(t)) \mathrm{d} x+\int_{\Gamma} \widehat{\beta}^{\varepsilon}\left(\rho_{\Gamma}(t)\right) \mathrm{d} \Gamma+\int_{0}^{t} \int_{\Omega}\left|\beta^{\varepsilon}(\rho)\right|^{2} \mathrm{~d} x \mathrm{~d} s \\
& +\int_{0}^{t} \int_{\Omega}\left(\beta^{\varepsilon}\right)^{\prime}(\rho)|\nabla \rho|^{2} \mathrm{~d} x \mathrm{~d} s+\int_{0}^{t} \int_{\Gamma}\left(\beta^{\varepsilon}\right)^{\prime}\left(\rho_{\Gamma}\right)\left|\nabla_{\Gamma} \rho_{\Gamma}\right|^{2} \mathrm{~d} \Gamma \mathrm{d} s \\
& =\int_{\Omega} \widehat{\beta}^{\varepsilon}\left(\rho_{0}\right) \mathrm{d} x+\int_{\Gamma} \widehat{\beta}^{\varepsilon}\left(\rho_{0_{\Gamma}}\right) \mathrm{d} \Gamma+\int_{0}^{t} \int_{\Omega}\left(\mathcal{T}_{\tau_{N}}(\mu) g^{\prime}(\rho)-\pi(\rho)\right) \beta^{\varepsilon}(\rho) \mathrm{d} x \mathrm{~d} s \\
& \quad+\int_{0}^{t} \int_{\Gamma}\left(u_{\Gamma}-\pi_{\Gamma}\left(\rho_{\Gamma}\right)\right) \beta^{\varepsilon}\left(\rho_{\Gamma}\right) \mathrm{d} \Gamma \mathrm{d} s-\int_{0}^{t} \int_{\Gamma} \beta_{\Gamma}^{\varepsilon}\left(\rho_{\Gamma}\right) \beta^{\varepsilon}\left(\rho_{\Gamma}\right) \mathrm{d} \Gamma \mathrm{d} s .
\end{aligned}
$$


In view of $(2.3)$, and as $\beta^{\varepsilon}$ is increasing, all of the terms on the left-hand side are nonnegative. About the last term, we exploit (3.2) to deduce that

$$
-\int_{0}^{t} \int_{\Gamma} \beta_{\Gamma}^{\varepsilon}\left(\rho_{\Gamma}\right) \beta^{\varepsilon}\left(\rho_{\Gamma}\right) \mathrm{d} \Gamma \mathrm{d} s \leq-\frac{1}{2 \eta} \int_{0}^{t} \int_{\Gamma}\left|\beta^{\varepsilon}\left(\rho_{\Gamma}\right)\right|^{2} \mathrm{~d} \Gamma \mathrm{d} s+\frac{T C_{\Gamma}^{2}}{2 \eta} \int_{\Gamma} 1 \mathrm{~d} \Gamma .
$$

Moreover, by (2.3) and (A6),

$$
\int_{\Omega} \widehat{\beta}^{\varepsilon}\left(\rho_{0}\right) \mathrm{d} x+\int_{\Gamma} \widehat{\beta}^{\varepsilon}\left(\rho_{0_{\Gamma}}\right) \mathrm{d} \Gamma \leq \int_{\Omega} \widehat{\beta}\left(\rho_{0}\right) \mathrm{d} x+\int_{\Gamma} \widehat{\beta}\left(\rho_{0_{\Gamma}}\right) \mathrm{d} \Gamma \leq K_{8} .
$$

Furthermore, thanks to the previous estimates and Young's inequality,

$$
\int_{0}^{t} \int_{\Omega}\left(\mathcal{T}_{\tau_{N}}(\mu) g^{\prime}(\rho)-\pi(\rho)\right) \beta^{\varepsilon}(\rho) \mathrm{d} x \mathrm{~d} s \leq \frac{1}{2} \int_{0}^{t} \int_{\Omega}\left|\beta^{\varepsilon}(\rho)\right|^{2} \mathrm{~d} x \mathrm{~d} s+K_{9},
$$

as well as

$$
\int_{0}^{t} \int_{\Gamma}\left(u_{\Gamma}-\pi_{\Gamma}\left(\rho_{\Gamma}\right)\right) \beta^{\varepsilon}\left(\rho_{\Gamma}\right) \mathrm{d} \Gamma \mathrm{d} s \leq \frac{1}{4 \eta} \int_{0}^{t} \int_{\Gamma}\left|\beta^{\varepsilon}\left(\rho_{\Gamma}\right)\right|^{2} \mathrm{~d} \Gamma \mathrm{d} s+K_{10},
$$

with the constant $\eta>0$ introduced in (A7). Hence, we can infer from (3.36)-(3.40) that

$$
\left\|\beta^{\varepsilon}\left(\rho_{\tau_{N}}^{\varepsilon}\right)\right\|_{L^{2}(0, T ; H)} \leq K_{11}
$$

whence, by comparison in (3.11),

$$
\left\|\Delta \rho_{\tau_{N}}^{\varepsilon}\right\|_{L^{2}(0, T ; H)} \leq K_{12}
$$

\section{FOURTH ESTIMATE:}

We now draw some consequences from (3.42). First, we invoke [3, Theorem 3.1, p. 1.79], which yields the estimate

$$
\int_{0}^{T}\|\rho(t)\|_{H^{3 / 2}(\Omega)}^{2} \mathrm{~d} t \leq K_{13} \int_{0}^{T}\left(\|\Delta \rho(t)\|_{H}^{2}+\left\|\rho_{\Gamma}(t)\right\|_{V_{\Gamma}}^{2}\right) \mathrm{d} t,
$$

whence we obtain that (cf. (3.35))

$$
\|\rho\|_{L^{2}\left(0, T ; H^{3 / 2}(\Omega)\right)} \leq K_{14} .
$$

By virtue of (3.42) and of [3, Theorem 2.27, p. 1.64], we can also conclude that

$$
\left\|\partial_{\mathbf{n}} \rho\right\|_{L^{2}\left(0, T ; H_{\Gamma}\right)} \leq K_{15} .
$$

We now aim to find an estimate for $\beta_{\Gamma}^{\varepsilon}\left(\rho_{\Gamma}\right)$. To this end, we multiply (3.12) by $\beta_{\Gamma}^{\varepsilon}\left(\rho_{\Gamma}\right)$ and integrate over $\Gamma \times(0, t)$, where $0<t \leq T$. We obtain the identity

$$
\begin{aligned}
& \int_{\Gamma} \widehat{\beta}_{\Gamma}^{\varepsilon}\left(\rho_{\Gamma}(t)\right) \mathrm{d} \Gamma+\int_{0}^{t} \int_{\Gamma}\left(\beta_{\Gamma}^{\varepsilon}\right)^{\prime}\left(\rho_{\Gamma}\right)\left|\nabla_{\Gamma} \rho_{\Gamma}\right|^{2} \mathrm{~d} \Gamma \mathrm{d} s+\int_{0}^{t} \int_{\Gamma}\left|\beta_{\Gamma}^{\varepsilon}\left(\rho_{\Gamma}\right)\right|^{2} \mathrm{~d} \Gamma \mathrm{d} s \\
& =\int_{\Gamma} \widehat{\beta}_{\Gamma}^{\varepsilon}\left(\rho_{0_{\Gamma}}\right) \mathrm{d} \Gamma+\int_{0}^{t} \int_{\Gamma}\left(u_{\Gamma}-\partial_{\mathbf{n}} \rho-\pi_{\Gamma}\left(\rho_{\Gamma}\right)\right) \beta_{\Gamma}^{\varepsilon}\left(\rho_{\Gamma}\right) \mathrm{d} \Gamma \mathrm{d} s .
\end{aligned}
$$


By the monotonicity of $\beta_{\Gamma}^{\varepsilon}$ and (2.3), the first two integrals on the left-hand side of (3.46) are nonnegative, while, thanks to (A6),

$$
\int_{\Gamma} \widehat{\beta}_{\Gamma}^{\varepsilon}\left(\rho_{0_{\Gamma}}\right) \mathrm{d} \Gamma \leq \int_{\Gamma} \widehat{\beta}_{\Gamma}\left(\rho_{0_{\Gamma}}\right) \mathrm{d} \Gamma \leq K_{16}
$$

For the last integral on the right-hand side, we invoke (A2), (A4), (3.35), (3.45), and Young's inequality, to infer that

$$
\int_{0}^{t} \int_{\Gamma}\left(u_{\Gamma}-\partial_{\mathbf{n}} \rho-\pi_{\Gamma}\left(\rho_{\Gamma}\right)\right) \beta_{\Gamma}^{\varepsilon}\left(\rho_{\Gamma}\right) \mathrm{d} \Gamma \mathrm{d} s \leq \frac{1}{2} \int_{0}^{t} \int_{\Gamma}\left|\beta_{\Gamma}^{\varepsilon}\left(\rho_{\Gamma}\right)\right|^{2} \mathrm{~d} \Gamma \mathrm{d} s+K_{17},
$$

whence we conclude that

$$
\left\|\beta_{\Gamma}^{\varepsilon}\left(\rho_{\Gamma}\right)\right\|_{L^{2}\left(0, T ; H_{\Gamma}\right)} \leq K_{18}
$$

Comparison in (3.12), using the previously shown estimates, then yields

$$
\left\|\Delta_{\Gamma} \rho_{\Gamma}\right\|_{L^{2}\left(0, T ; H_{\Gamma}\right)} \leq K_{19}
$$

and it follows from the boundary version of the elliptic estimates that

$$
\left\|\rho_{\Gamma}\right\|_{L^{2}\left(0, T ; H^{2}(\Gamma)\right)} \leq K_{20}
$$

whence, employing (3.42) and standard elliptic estimates, we conclude that also

$$
\|\rho\|_{L^{2}\left(0, T ; H^{2}(\Omega)\right)} \leq K_{21} .
$$

In conclusion, we have inferred the estimate

$$
\left\|\beta_{\Gamma}^{\varepsilon}\left(\rho_{\tau_{N_{\Gamma}}}^{\varepsilon}\right)\right\|_{L^{2}\left(0, T ; H_{\Gamma}\right)}+\left\|\rho_{\tau_{N}}^{\varepsilon}\right\|_{L^{2}\left(0, T ; H^{2}(\Omega)\right)}+\left\|\rho_{\tau_{N_{\Gamma}}^{\varepsilon}}\right\|_{L^{2}\left(0, T ; H^{2}(\Gamma)\right)} \leq K_{22} .
$$

\section{FifTh ESTimate:}

In this step of the proof, we partly repeat a formal argument that was already used in Step 1, noting again that it can be made rigorous by using finite differences in time. Namely, we differentiate (3.11) formally with respect to time, multiply the resulting identity by $\partial_{t} \rho$, and integrate over $Q_{t}$, where $0<t \leq T$, and (formally) by parts. As in Step 1 (see the argumentation between Eqs. (3.24) and (3.27)), we then arrive at an inequality of the form

$$
\begin{aligned}
& \frac{1}{2}\left(\left\|\partial_{t} \rho(t)\right\|_{H}^{2}+\left\|\partial_{t} \rho_{\Gamma}(t)\right\|_{H_{\Gamma}}^{2}\right)+\int_{0}^{t} \int_{\Omega}\left|\nabla \partial_{t} \rho\right|^{2} \mathrm{~d} x \mathrm{~d} s+\int_{0}^{t} \int_{\Gamma}\left|\nabla_{\Gamma} \partial_{t} \rho_{\Gamma}\right|^{2} \mathrm{~d} \Gamma \mathrm{d} s \\
& \leq K_{23}+\sum_{j=1}^{4} I_{j},
\end{aligned}
$$

where the expressions $I_{j}, 1 \leq j \leq 4$, will be specified and estimated below. At first, recall that $\pi^{\prime}, \pi_{\Gamma}^{\prime}, g^{\prime}, g^{\prime \prime}$ are bounded. Hence, owing to (3.35), we have that

$$
I_{1}:=-\int_{0}^{t} \int_{\Omega} \pi^{\prime}(\rho)\left|\partial_{t} \rho\right|^{2} \mathrm{~d} x \mathrm{~d} s \leq K_{24}
$$


and, by also using (A2) and Young's inequality, we infer that

$$
I_{4}:=\int_{0}^{t} \int_{\Gamma}\left(\partial_{t} u_{\Gamma}-\pi_{\Gamma}^{\prime}\left(\rho_{\Gamma}\right) \partial_{t} \rho_{\Gamma}\right) \partial_{t} \rho_{\Gamma} \mathrm{d} \Gamma \mathrm{d} s \leq K_{25}
$$

In addition, invoking (3.31) and the compactness inequality (1.21), as well as Hölder's inequality, we find that

$$
\begin{aligned}
I_{2}:= & \int_{0}^{t} \int_{\Omega} \mathcal{T}_{\tau_{N}}(\mu) g^{\prime \prime}(\rho)\left|\partial_{t} \rho\right|^{2} \mathrm{~d} x \mathrm{~d} s \leq K_{26} \int_{0}^{t}\left\|\mathcal{T}_{\tau_{N}}(\mu(s))\right\|_{2}\left\|\partial_{t} \rho(s)\right\|_{4}^{2} \mathrm{~d} s \\
& \leq K_{27} \int_{0}^{t} \| \partial_{t}\left(\rho(s)\left\|_{4}^{2} \mathrm{~d} s \leq \frac{1}{6} \int_{0}^{t}\right\| \nabla \partial_{t} \rho(s) \|_{H}^{2} \mathrm{~d} s+K_{28} .\right.
\end{aligned}
$$

The estimation of the remaining term

$$
I_{3}:=\int_{0}^{t} \int_{\Omega} \partial_{t} \mathcal{T}_{\tau_{N}}(\mu) g^{\prime}(\rho) \partial_{t} \rho \mathrm{d} x \mathrm{~d} s
$$

is more delicate. To this end, we note that (3.9) implies the identity

$$
\partial_{t} \mu=\frac{1}{1+2 g(\rho)}\left(\Delta \mu-\mu g^{\prime}(\rho) \partial_{t} \rho\right)
$$

where, thanks to $(3.3), 1 /(1+2 g(\rho)) \leq 3$. First, recall that $\mathcal{T}_{\tau_{N}}(\mu)$ is constant with respect to time on the interval $(0, \tau)$. We therefore have, with obvious notation:

$$
\begin{aligned}
& \int_{0}^{t} \int_{\Omega} g^{\prime}(\rho) \partial_{t} \mathcal{T}_{\tau_{N}}(\mu) \partial_{t} \rho \mathrm{d} x \mathrm{~d} s=\int_{0}^{t-\tau_{N}} \int_{\Omega} \partial_{t} \mu(s) g^{\prime}\left(\rho\left(s+\tau_{N}\right)\right) \partial_{t} \rho\left(s+\tau_{N}\right) \mathrm{d} x \mathrm{~d} s \\
& =\int_{0}^{t-\tau_{N}} \int_{\Omega} \frac{1}{1+2 g(\rho(s))}\left[\Delta \mu(s)-\mu(s) g^{\prime}(\rho(s)) \partial_{t} \rho(s)\right] \partial_{t} g\left(\rho\left(s+\tau_{N}\right)\right) \mathrm{d} x \mathrm{~d} s \\
& =-\int_{0}^{t-\tau_{N}} \int_{\Omega} \nabla \mu(s) \cdot \nabla\left(\frac{\partial_{t} g\left(\rho\left(s+\tau_{N}\right)\right)}{1+2 g(\rho(s))}\right) \mathrm{d} x \mathrm{~d} s \\
& \quad-\int_{0}^{t-\tau_{N}} \int_{\Omega} \frac{g^{\prime}(\rho(s)) g^{\prime}\left(\rho\left(s+\tau_{N}\right)\right)}{1+2 g(\rho(s))} \mu(s) \partial_{t} \rho(s) \partial_{t} \rho\left(s+\tau_{N}\right) \mathrm{d} x \mathrm{~d} s
\end{aligned}
$$

We treat the last two integrals separately, invoking our structural assumptions and the estimates established above. We have

$$
\begin{aligned}
& -\int_{0}^{t-\tau_{N}} \int_{\Omega} \nabla \mu(s) \cdot \nabla\left(\frac{\partial_{t} g\left(\rho\left(s+\tau_{N}\right)\right)}{1+2 g(\rho(s))}\right) \mathrm{d} x \mathrm{~d} s \\
= & -\int_{0}^{t-\tau_{N}} \int_{\Omega} \nabla \mu(s) \cdot \nabla\left(\frac{g^{\prime}\left(\rho\left(s+\tau_{N}\right)\right) \partial_{t} \rho\left(s+\tau_{N}\right)}{1+2 g(\rho(s))}\right) \mathrm{d} x \mathrm{~d} s \\
\leq & K_{29} \int_{0}^{t-\tau_{N}} \int_{\Omega}|\nabla \mu(s)|\left|\nabla \partial_{t} \rho\left(s+\tau_{N}\right)\right| \mathrm{d} x \mathrm{~d} s \\
& +K_{30} \int_{0}^{t-\tau_{N}} \int_{\Omega}|\nabla \mu(s)||\nabla \rho(s)|\left|\partial_{t} \rho\left(s+\tau_{N}\right)\right| \mathrm{d} x \mathrm{~d} s \\
& +K_{31} \int_{0}^{t-\tau_{N}} \int_{\Omega}|\nabla \mu(s)|\left|\nabla \rho\left(s+\tau_{N}\right)\right|\left|\partial_{t} \rho\left(s+\tau_{N}\right)\right| \mathrm{d} x \mathrm{~d} s,
\end{aligned}
$$


where, owing to (3.30) and Young's inequality,

$$
K_{29} \int_{0}^{t-\tau_{N}} \int_{\Omega}|\nabla \mu(s)|\left|\nabla \partial_{t} \rho\left(s+\tau_{N}\right)\right| \mathrm{d} x \mathrm{~d} s \leq \frac{1}{6} \int_{0}^{t} \int_{\Omega}\left|\nabla \partial_{t} \rho\right|^{2} \mathrm{~d} x \mathrm{~d} s+K_{32} .
$$

On the other hand, we also have that

$$
\begin{aligned}
& K_{30} \int_{0}^{t-\tau_{N}} \int_{\Omega}|\nabla \mu(s)||\nabla \rho(s)|\left|\partial_{t} \rho\left(s+\tau_{N}\right)\right| \mathrm{d} x \mathrm{~d} s \\
& \leq K_{30} \int_{0}^{t-\tau_{N}}\|\nabla \mu(s)\|_{2}\|\nabla \rho(s)\|_{4}\left\|\partial_{t} \rho\left(s+\tau_{N}\right)\right\|_{4} \mathrm{~d} s \\
& \leq \frac{1}{6} \int_{0}^{t}\left\|\partial_{t} \rho(s)\right\|_{V}^{2} \mathrm{~d} s+K_{33} \int_{0}^{t-\tau_{N}}\|\nabla \mu(s)\|_{H}^{2}\|\nabla \rho(s)\|_{V}^{2} \mathrm{~d} s \\
& \leq \frac{1}{6} \int_{0}^{t} \int_{\Omega}\left|\nabla \partial_{t} \rho\right|^{2} \mathrm{~d} x \mathrm{~d} s+K_{34} \int_{0}^{t} \int_{\Omega}\left|\partial_{t} \rho\right|^{2} \mathrm{~d} x \mathrm{~d} s+K_{35} \int_{0}^{t-\tau_{N}}\|\nabla \mu(s)\|_{H}^{2}\|\nabla \rho(s)\|_{V}^{2} \mathrm{~d} s \\
& \leq \frac{1}{6} \int_{0}^{t} \int_{\Omega}\left|\nabla \partial_{t} \rho\right|^{2} \mathrm{~d} x \mathrm{~d} s+K_{36}+K_{37} \int_{0}^{t-\tau_{N}}\|\nabla \mu(s)\|_{H}^{2}\|\nabla \rho(s)\|_{V}^{2} \mathrm{~d} s .
\end{aligned}
$$

The last integral cannot be controlled in this form. We thus try to estimate the expression $\|\nabla \rho(s)\|_{V}^{2}$ in terms of the expressions $\left\|\partial_{t} \rho(s)\right\|_{H}^{2}$ and $\left\|\partial_{t} \rho_{\Gamma}(s)\right\|_{H_{\Gamma}}^{2}$ which can be handled using the first summand on the left-hand side of (3.54). To this end, we use the regularity theory for linear elliptic equations and (3.35) to deduce that

$$
\|\nabla \rho(s)\|_{V}^{2} \leq K_{38}\left(\|\rho(s)\|_{V}^{2}+\|\Delta \rho(s)\|_{H}^{2}\right) \leq K_{39}\left(1+\|\Delta \rho(s)\|_{H}^{2}\right) .
$$

We now multiply, just as in the third estimate above, (3.11) by $\beta^{\varepsilon}(\rho(s)$ ), but this time we only integrate over $\Omega$ to obtain the identity (compare with (3.36))

$$
\begin{aligned}
& \left\|\beta^{\varepsilon}(\rho(s))\right\|_{H}^{2}+\int_{\Omega}\left(\beta^{\varepsilon}\right)^{\prime}(\rho(s))|\nabla \rho(s)|^{2} \mathrm{~d} x+\int_{\Gamma}\left(\beta^{\varepsilon}\right)^{\prime}\left(\rho_{\Gamma}(s)\right)\left|\nabla_{\Gamma} \rho_{\Gamma}(s)\right|^{2} \mathrm{~d} \Gamma \\
& =\int_{\Omega} \beta^{\varepsilon}(\rho(s))\left(-\partial_{t} \rho(s)-\pi(\rho(s))+\mathcal{T}_{\tau_{N}}(\mu(s)) g^{\prime}(\rho(s))\right) \mathrm{d} x \\
& \quad+\int_{\Gamma} \beta^{\varepsilon}\left(\rho_{\Gamma}(s)\right)\left(u_{\Gamma}(s)-\pi_{\Gamma}\left(\rho_{\Gamma}(s)\right)-\partial_{t} \rho_{\Gamma}(s)\right) \mathrm{d} \Gamma-\int_{\Gamma} \beta_{\Gamma}^{\varepsilon}\left(\rho_{\Gamma}(s)\right) \beta^{\varepsilon}\left(\rho_{\Gamma}(s)\right) \mathrm{d} \Gamma .
\end{aligned}
$$

The terms on the left-hand side are nonnegative, and analogous reasoning as in the third estimate, using the already proven bounds, the general assumptions, (3.31), (3.2) and Young's inequality, shows that

$$
\left\|\beta^{\varepsilon}(\rho(s))\right\|_{H}^{2} \leq K_{40}\left(1+\left\|\partial_{t} \rho(s)\right\|_{H}^{2}+\left\|\partial_{t} \rho_{\Gamma}(s)\right\|_{H_{\Gamma}}^{2}\right) \quad \text { for a. e. } s \in(0, t),
$$

whence, by comparison in (3.11),

$$
\|\Delta \rho(s)\|_{H}^{2} \leq K_{41}\left(1+\left\|\partial_{t} \rho(s)\right\|_{H}^{2}+\left\|\partial_{t} \rho_{\Gamma}(s)\right\|_{H_{\Gamma}}^{2}\right) \quad \text { for a.e. } s \in(0, t) .
$$

Combining the estimates (3.62)-(3.66), we have thus shown that

$$
\begin{aligned}
& \int_{0}^{t-\tau_{N}} \int_{\Omega}|\nabla \mu(s)||\nabla \rho(s)|\left|\partial_{t} \rho\left(s+\tau_{N}\right)\right| \mathrm{d} x \mathrm{~d} s \\
& \leq \frac{1}{6} \int_{0}^{t} \int_{\Omega}\left|\nabla \partial_{t} \rho\right|^{2} \mathrm{~d} x \mathrm{~d} s+K_{42}\left(1+\int_{0}^{t}\|\nabla \mu(s)\|_{H}^{2}\left(\left\|\partial_{t} \rho(s)\right\|_{H}^{2}+\left\|\partial_{t} \rho_{\Gamma}(s)\right\|_{H_{\Gamma}}^{2}\right) \mathrm{d} s\right) .
\end{aligned}
$$


By similar reasoning, we also obtain that

$$
\begin{aligned}
K_{31} \int_{0}^{t-\tau_{N}} \int_{\Omega}|\nabla \mu(s)|\left|\nabla \rho\left(s+\tau_{N}\right)\right|\left|\partial_{t} \rho\left(s+\tau_{N}\right)\right| \mathrm{d} x \mathrm{~d} s \leq \frac{1}{6} \int_{0}^{t} \int_{\Omega}\left|\nabla \partial_{t} \rho\right|^{2} \mathrm{~d} x \mathrm{~d} s \\
+K_{43}\left(1+\int_{0}^{t}\left\|\nabla \mathcal{T}_{\tau_{N}}(\mu(s))\right\|_{H}^{2}\left(\left\|\partial_{t} \rho(s)\right\|_{H}^{2}+\left\|\partial_{t} \rho_{\Gamma}(s)\right\|_{H_{\Gamma}}^{2}\right) \mathrm{d} s\right),
\end{aligned}
$$

where $\nabla \mathcal{T}_{\tau_{N}}(\mu(s))=\nabla \mu_{0}$ for $0 \leq s \leq \tau_{N}$. Hence, combining the inequalities (3.60)(3.61) and (3.67)-(3.68), we have proved the estimate

$$
\begin{aligned}
& -\int_{0}^{t-\tau_{N}} \int_{\Omega} \nabla \mu(s) \cdot \nabla\left(\frac{\partial_{t} g\left(\rho\left(s+\tau_{N}\right)\right)}{1+2 g(\rho(s))}\right) \mathrm{d} x \mathrm{~d} s \leq \frac{1}{2} \int_{0}^{t} \int_{\Omega}\left|\nabla \partial_{t} \rho\right|^{2} \mathrm{~d} x \mathrm{~d} s \\
& +K_{44}\left(1+\int_{0}^{t}\left(\|\nabla \mu(s)\|_{H}^{2}+\left\|\nabla \mathcal{T}_{\tau_{N}}(\mu(s))\right\|_{H}^{2}\right)\left(\left\|\partial_{t} \rho(s)\right\|_{H}^{2}+\left\|\partial_{t} \rho_{\Gamma}(s)\right\|_{H_{\Gamma}}^{2}\right) \mathrm{d} s\right) .
\end{aligned}
$$

Let us now come to the second term on the right-hand side of (3.59). We have, owing to Hölder's and Young's inequalities,

$$
\begin{aligned}
& -\int_{0}^{t-\tau_{N}} \int_{\Omega} \frac{g^{\prime}(\rho(s)) g^{\prime}\left(\rho\left(s+\tau_{N}\right)\right)}{1+2 g(\rho(s))} \mu(s) \partial_{t} \rho(s) \partial_{t} \rho\left(s+\tau_{N}\right) \mathrm{d} x \mathrm{~d} s \\
& \leq K_{45} \int_{0}^{t-\tau_{N}}\|\mu(s)\|_{3}\left\|\partial_{t} \rho\left(s+\tau_{N}\right)\right\|_{6}\left\|\partial_{t} \rho(s)\right\|_{2} \mathrm{~d} s \\
& \leq \frac{1}{6} \int_{0}^{t-\tau_{N}}\left\|\partial_{t} \rho\left(s+\tau_{N}\right)\right\|_{V}^{2} \mathrm{~d} s+K_{46} \int_{0}^{t}\|\mu(s)\|_{3}^{2}\left\|\partial_{t} \rho(s)\right\|_{H}^{2} \mathrm{~d} s \\
& \leq \frac{1}{6} \int_{0}^{t}\left\|\nabla \partial_{t} \rho(s)\right\|_{H}^{2} \mathrm{~d} s+K_{47}\left(1+\int_{0}^{t}\|\mu(s)\|_{3}^{2}\left\|\partial_{t} \rho(s)\right\|_{H}^{2} \mathrm{~d} s\right) .
\end{aligned}
$$

Therefore, due to (3.69) and (3.70), we can infer from (3.59) that

$$
\begin{aligned}
& \int_{0}^{t} \int_{\Omega} g^{\prime}(\rho) \partial_{t} \mathcal{\tau}_{\tau_{N}}(\mu) \partial_{t} \rho \mathrm{d} x \mathrm{~d} s \leq \frac{2}{3} \int_{0}^{t} \int_{\Omega}\left|\nabla \partial_{t} \rho\right|^{2} \mathrm{~d} x \mathrm{~d} s+K_{48} \\
& +K_{49} \int_{0}^{t} \Phi(s)\left(\left\|\partial_{t} \rho(s)\right\|_{H}^{2}+\left\|\partial_{t} \rho_{\Gamma}(s)\right\|_{H_{\Gamma}}^{2}\right) \mathrm{d} s,
\end{aligned}
$$

where $\Phi:(0, T) \rightarrow \mathbb{R}$ is given by $\Phi(s):=\|\mu(s)\|_{3}^{2}+\|\nabla \mu(s)\|_{H}^{2}+\left\|\nabla \mathcal{T}_{\tau_{N}}(\mu(s))\right\|_{H}^{2}$ for $s \in(0, T)$. Since it is known that $\Phi$ is bounded in $L^{1}(0, T)$ uniformly with respect to $N \in \mathbb{N}$ and $\varepsilon>0$, summarizing the estimates (3.54)-(3.57), (3.71), and invoking Gronwall's lemma, we have thus finally shown that

$$
\left\|\partial_{t} \rho_{\tau_{N}}^{\varepsilon}\right\|_{L^{\infty}(0, T ; H) \cap L^{2}(0, T ; V)}+\left\|\partial_{t} \rho_{\tau_{N_{\Gamma}}^{\varepsilon}}^{\varepsilon}\right\|_{L^{\infty}\left(0, T ; H_{\Gamma}\right) \cap L^{2}\left(0, T ; V_{\Gamma}\right)} \leq K_{50} .
$$

Let us draw some consequences from this estimate. First note that (3.65), (3.66) and (3.72) entail that

$$
\left\|\beta^{\varepsilon}\left(\rho_{\tau_{N}}^{\varepsilon}\right)\right\|_{L^{\infty}(0, T ; H)}+\left\|\Delta \rho_{\tau_{N}}^{\varepsilon}\right\|_{L^{\infty}(0, T ; H)} \leq K_{51} .
$$


Next, we recall (3.35) and the fact that $u_{\Gamma} \in C^{0}\left([0, T] ; H_{\Gamma}\right)$, by assumption (A2). We therefore can, for almost every fixed $t \in(0, T)$, follow exactly the same chain of arguments as in the fourth estimate, but this time without integrating over time. In doing this, we establish consecutively bounds resembling the estimates (3.43)-(3.53), eventually arriving at the estimate

$$
\left\|\beta_{\Gamma}^{\varepsilon}\left(\rho_{\tau_{N_{\Gamma}}}^{\varepsilon}\right)\right\|_{L^{\infty}\left(0, T ; H_{\Gamma}\right)}+\left\|\rho_{\tau_{N}}^{\varepsilon}\right\|_{L^{\infty}\left(0, T ; H^{2}(\Omega)\right)}+\left\|\rho_{\tau_{N_{\Gamma}}}^{\varepsilon}\right\|_{L^{\infty}\left(0, T ; H^{2}(\Gamma)\right)} \leq K_{52}
$$

Notice that (3.74) implies, in particular, that

$$
\left\|\rho_{\tau_{N}}^{\varepsilon}\right\|_{L^{\infty}(Q)}+\left\|\rho_{\tau_{N_{\Gamma}}}^{\varepsilon}\right\|_{L^{\infty}(\Sigma)} \leq K_{53}
$$

\section{SiXth ESTIMATE:}

We now multiply (3.9) by $\partial_{t} \mu$ and integrate over $Q_{t}$, where $0<t \leq T$, and by parts. Recalling (3.3) and (A1), and invoking Hölder's and Young's inequalities, we obtain that

$$
\begin{aligned}
& \frac{1}{3} \int_{0}^{t} \int_{\Omega}\left|\partial_{t} \mu\right|^{2} \mathrm{~d} x \mathrm{~d} s+\frac{1}{2}\|\nabla \mu(t)\|_{H}^{2} \leq \frac{1}{2}\left\|\nabla \mu_{0}\right\|_{H}^{2}+K_{54} \int_{0}^{t} \int_{\Omega}\left|\partial_{t} \mu\right||\mu|\left|\partial_{t} \rho\right| \mathrm{d} x \mathrm{~d} s \\
& \leq K_{55}+K_{56} \int_{0}^{t}\left\|\partial_{t} \mu(s)\right\|_{2}\left\|\partial_{t} \rho(s)\right\|_{4}\|\mu(s)\|_{4} \mathrm{~d} s \\
& \leq K_{55}+\frac{1}{6} \int_{0}^{t} \int_{\Omega}\left|\partial_{t} \mu\right|^{2} \mathrm{~d} x \mathrm{~d} s+K_{57} \int_{0}^{t}\left\|\partial_{t} \rho(s)\right\|_{V}^{2}\|\mu(s)\|_{V}^{2} \mathrm{~d} s
\end{aligned}
$$

and we note that the function $s \mapsto\left\|\partial_{t} \rho(s)\right\|_{V}^{2}$ is bounded in $L^{1}(0, T)$ by (3.72). Thus, taking (3.30) into account, we can infer from Gronwall's lemma that

$$
\left\|\mu_{\tau_{N}}^{\varepsilon}\right\|_{H^{1}(0, T ; H) \cap L^{\infty}(0, T ; V)} \leq K_{58} .
$$

But then, owing to Hölder's inequality and and (3.72),

$$
\begin{aligned}
& \left\|g^{\prime}(\rho) \partial_{t} \rho \mu\right\|_{L^{2}(Q)}^{2} \leq K_{59} \int_{0}^{T}\left\|\partial_{t} \rho(s)\right\|_{4}^{2}\|\mu(s)\|_{4}^{2} \mathrm{~d} s \\
& \leq K_{60}\|\mu\|_{L^{\infty}(0, T ; V)}^{2}\left\|\partial_{t} \rho\right\|_{L^{2}(0, T ; V)}^{2} \leq K_{61},
\end{aligned}
$$

whence, by comparison in (3.9),

$$
\|\Delta \mu\|_{L^{2}(0, T ; H)} \leq K_{62}
$$

In view of the boundary condition (3.10), we therefore can infer from standard elliptic estimates that

$$
\left\|\mu_{\tau_{N}}^{\varepsilon}\right\|_{L^{2}\left(0, T ; H^{2}(\Omega)\right)} \leq K_{63}
$$

Finally, we recall (3.72) and (3.28), which yield that $\partial_{t} \rho_{\tau_{N}}^{\varepsilon}$ is bounded in the space $L^{7 / 3}\left(0, T ; L^{14 / 3}(\Omega)\right)$, uniformly in $N \in \mathbb{N}$ and $\varepsilon>0$. Hence, we may again argue as in the proof of $[14$, Thm. 2.3] to conclude that

$$
\left\|\mu_{\tau_{N}}^{\varepsilon}\right\|_{L^{\infty}(Q)} \leq K_{64}
$$


STEP 3: In this step of the proof, we perform the limit process as $N \rightarrow \infty$. Owing to the a priori estimates established above in Step 2, we can select a subsequence, which is again indexed by $N$, such that, as $N \rightarrow \infty$,

$$
\begin{aligned}
& \rho_{\tau_{N}}^{\varepsilon} \rightarrow \rho^{\varepsilon} \quad \text { weakly-star in } W^{1, \infty}(0, T ; H) \cap H^{1}(0, T ; V) \cap L^{\infty}\left(0, T ; H^{2}(\Omega)\right), \\
& \rho_{\tau_{N_{\Gamma}}}^{\varepsilon} \rightarrow \rho_{\Gamma}^{\varepsilon} \quad \text { weakly-star in } W^{1, \infty}\left(0, T ; H_{\Gamma}\right) \cap H^{1}\left(0, T ; V_{\Gamma}\right) \cap L^{\infty}\left(0, T ; H^{2}(\Gamma)\right), \\
& \mu_{\tau_{N}}^{\varepsilon} \rightarrow \mu^{\varepsilon} \quad \text { weakly in } H^{1}(0, T ; H) \cap L^{2}(0, T ; W) \text { and weakly-star in } L^{\infty}(Q),
\end{aligned}
$$

for suitable limits $\mu^{\varepsilon}, \rho^{\varepsilon}, \rho_{\Gamma}^{\varepsilon}$. By (3.82), we have that $\partial_{\mathbf{n}} \mu^{\varepsilon}=0$ almost everwhere on $\Sigma$, and the continuity of the embedding $H^{1}(0, T ; H) \cap L^{2}\left(0, T ; H^{2}(\Omega)\right) \subset C^{0}([0, T] ; V)$ implies that both $\rho^{\varepsilon}(0)=\rho_{0}$ and $\mu^{\varepsilon}(0)=\mu_{0}$. Moreover, by virtue of standard compactness results (see, e. g., [45, Sect. 8, Cor. 4]), we can without loss of generality assume that

$$
\begin{array}{ll}
\mu_{\tau_{N}}^{\varepsilon} \rightarrow \mu^{\varepsilon} & \text { strongly in } C^{0}([0, T] ; H), \\
\rho_{\tau_{N}}^{\varepsilon} \rightarrow \rho^{\varepsilon} & \text { strongly in } C^{0}\left([0, T] ; H^{s}(\Omega)\right) \text { for } 0<s<2,
\end{array}
$$

whence we obtain that $\mu^{\varepsilon} \geq 0$ almost everywhere in $Q$ and

$$
\rho_{\tau_{N}}^{\varepsilon} \rightarrow \rho^{\varepsilon} \quad \text { strongly in } C^{0}(\bar{Q}) .
$$

But then $\rho_{\tau_{N} \mid \Sigma}^{\varepsilon} \rightarrow \rho_{\mid \Sigma}^{\varepsilon}$ strongly in $C^{0}(\bar{\Sigma})$, and thus, invoking (3.81), we obtain that $\rho_{\mid \Sigma}^{\varepsilon}=\rho_{\Gamma}^{\varepsilon}$. In addition, the Lipschitz continuity of the corresponding functions on $\mathbb{R}$ yields that

$$
\begin{aligned}
& \Phi\left(\rho_{\tau_{N}}^{\varepsilon}\right) \rightarrow \Phi\left(\rho^{\varepsilon}\right) \text { strongly in } C^{0}(\bar{Q}) \text { for } \Phi \in\left\{\beta^{\varepsilon}, \pi, g, g^{\prime}\right\} \\
& \Phi_{\Gamma}\left(\rho_{\tau_{N_{\Gamma}}}^{\varepsilon}\right) \rightarrow \Phi_{\Gamma}\left(\rho_{\Gamma}^{\varepsilon}\right) \text { strongly in } C^{0}(\bar{\Sigma}) \text { for } \Phi_{\Gamma} \in\left\{\beta_{\Gamma}^{\varepsilon}, \pi_{\Gamma}\right\}
\end{aligned}
$$

It is therefore easily verified that

$$
\begin{aligned}
& \left(1+2 g\left(\rho_{\tau_{N}}^{\varepsilon}\right)\right) \partial_{t} \mu_{\tau_{N}}^{\varepsilon} \rightarrow\left(1+2 g\left(\rho^{\varepsilon}\right)\right) \partial_{t} \mu^{\varepsilon} \quad \text { weakly in } L^{2}(Q), \\
& \mu_{\tau_{N}}^{\varepsilon} g^{\prime}\left(\rho_{\tau_{N}}^{\varepsilon}\right) \partial_{t} \rho_{\tau_{N}}^{\varepsilon} \rightarrow \mu^{\varepsilon} g^{\prime}\left(\rho^{\varepsilon}\right) \partial_{t} \rho^{\varepsilon} \quad \text { weakly in } L^{1}(Q) .
\end{aligned}
$$

Finally, in view of (3.77), it is easy to check that

$$
\left\|\mathcal{T}_{\tau_{N}}\left(\mu_{\tau_{N}}^{\varepsilon}\right)-\mu_{\tau_{N}}^{\varepsilon}\right\|_{L^{2}(Q)} \rightarrow 0 \text { as } N \rightarrow \infty
$$

which, in combination with (3.83) and (3.86), implies that

$$
\mathcal{T}_{\tau_{N}}\left(\mu_{\tau_{N}}^{\varepsilon}\right) g^{\prime}\left(\rho_{\tau_{N}}^{\varepsilon}\right) \rightarrow \mu^{\varepsilon} g^{\prime}\left(\rho^{\varepsilon}\right) \text { strongly in } L^{2}(Q) .
$$

Hence, letting $N \rightarrow \infty$ in the system (3.9)-(3.13), we find that the triple $\left(\mu^{\varepsilon}, \rho^{\varepsilon}, \rho_{\Gamma}^{\varepsilon}\right)$ is a solution to the initial-boundary value problem

$$
\begin{aligned}
& \left(1+2 g\left(\rho^{\varepsilon}\right)\right) \partial_{t} \mu^{\varepsilon}+g^{\prime}\left(\rho^{\varepsilon}\right) \partial_{t} \rho^{\varepsilon} \mu^{\varepsilon}-\Delta \mu^{\varepsilon}=0 \quad \text { a.e. in } Q, \\
& \partial_{\mathbf{n}} \mu^{\varepsilon}=0 \quad \text { a.e. on } \Sigma, \\
& \partial_{t} \rho^{\varepsilon}-\Delta \rho^{\varepsilon}+\beta^{\varepsilon}\left(\rho^{\varepsilon}\right)+\pi\left(\rho^{\varepsilon}\right)=\mu^{\varepsilon} g^{\prime}\left(\rho^{\varepsilon}\right) \quad \text { a. e. in } Q, \\
& \partial_{\mathbf{n}} \rho^{\varepsilon}+\partial_{t} \rho_{\Gamma}^{\varepsilon}+\beta_{\Gamma}^{\varepsilon}\left(\rho_{\Gamma}^{\varepsilon}\right)+\pi_{\Gamma}\left(\rho_{\Gamma}^{\varepsilon}\right)-\Delta_{\Gamma} \rho_{\Gamma}^{\varepsilon}=u_{\Gamma}, \quad \rho_{\Gamma}^{\varepsilon}=\rho_{\mid \Sigma}^{\varepsilon}, \quad \text { a.e. on } \Sigma, \\
& \mu^{\varepsilon}(0)=\mu_{0} \quad \text { and } \quad \rho^{\varepsilon}(0)=\rho_{0} \quad \text { a.e. in } \Omega, \quad \rho_{\Gamma}^{\varepsilon}(0)=\rho_{0 \mid \Gamma} \quad \text { a.e. on } \Gamma \text {. }
\end{aligned}
$$


Moreover, $\mu^{\varepsilon}$ is nonnegative almost everywhere in $Q$, and, by virtue of the weak (and weak star) sequential lower semicontinuity of norms, we have the estimate

$$
\begin{aligned}
& \left\|\rho^{\varepsilon}\right\|_{W^{1, \infty}(0, T ; H) \cap H^{1}(0, T ; V) \cap L^{\infty}\left(0, T ; H^{2}(\Omega)\right)} \\
& +\left\|\rho_{\Gamma}^{\varepsilon}\right\|_{W^{1, \infty}\left(0, T ; H_{\Gamma}\right) \cap H^{1}\left(0, T ; V_{\Gamma}\right) \cap L^{\infty}\left(0, T ; H^{2}(\Gamma)\right)} \\
& +\left\|\mu^{\varepsilon}\right\|_{H^{1}(0, T ; H) \cap L^{2}(0, T ; W) \cap L^{\infty}(Q)} \\
& +\left\|\beta^{\varepsilon}\left(\rho^{\varepsilon}\right)\right\|_{L^{\infty}(0, T ; H)}+\left\|\beta_{\Gamma}^{\varepsilon}\left(\rho_{\Gamma}^{\varepsilon}\right)\right\|_{L^{\infty}\left(0, T ; H_{\Gamma}\right)} \leq K_{65} .
\end{aligned}
$$

STEP 4: In the final step of the existence proof, we perform the limit process as $\varepsilon \searrow 0$. Repeating the arguments of Step 3, we can find a sequence $\varepsilon_{n} \searrow 0$ and corresponding solution triples $\left(\mu^{\varepsilon_{n}}, \rho^{\varepsilon_{n}}, \rho_{\Gamma}^{\varepsilon_{n}}\right)$ to the system (3.89)-(3.93) such that, as $n \rightarrow \infty$,

$$
\begin{aligned}
& \rho^{\varepsilon_{n}} \rightarrow \rho \quad \text { weakly-star in } W^{1, \infty}(0, T ; H) \cap H^{1}(0, T ; V) \cap L^{\infty}\left(0, T ; H^{2}(\Omega)\right), \\
& \rho_{\Gamma}^{\varepsilon_{n}} \rightarrow \rho_{\Gamma} \quad \text { weakly-star in } W^{1, \infty}\left(0, T ; H_{\Gamma}\right) \cap H^{1}\left(0, T ; V_{\Gamma}\right) \cap L^{\infty}\left(0, T ; H^{2}(\Gamma)\right), \\
& \mu^{\varepsilon_{n}} \rightarrow \mu \quad \text { weakly in } H^{1}(0, T ; H) \cap L^{2}(0, T ; W) \text { and weakly-star in } L^{\infty}(Q), \\
& \beta^{\varepsilon_{n}}\left(\rho^{\varepsilon_{n}}\right) \rightarrow \xi \quad \text { weakly-star in } L^{\infty}(0, T ; H), \\
& \beta_{\Gamma}^{\varepsilon_{n}}\left(\rho_{\Gamma}^{\varepsilon_{n}}\right) \rightarrow \xi_{\Gamma} \quad \text { weakly-star in } L^{\infty}\left(0, T ; H_{\Gamma}\right),
\end{aligned}
$$

for suitable limits $\mu, \rho, \rho_{\Gamma}, \xi, \xi_{\Gamma}$. As in Step 3, we may assume that

$$
\mu^{\varepsilon_{n}} \rightarrow \mu \text { strongly in } C^{0}([0, T] ; H), \quad \rho^{\varepsilon_{n}} \rightarrow \rho \text { strongly in } C^{0}(\bar{Q}),
$$

whence we again conclude that $\rho_{\Gamma}=\rho_{\mid \Sigma}, \rho(0)=\rho_{0}$, and $\mu(0)=\mu_{0}$. Moreover, (3.97) implies that $\partial_{\mathbf{n}} \mu=0$ almost everywhere on $\Sigma$. We also have that

$$
\begin{aligned}
& \Phi\left(\rho^{\varepsilon_{n}}\right) \rightarrow \Phi(\rho) \quad \text { strongly in } C^{0}(\bar{Q}) \text { for } \Phi \in\left\{\pi, g, g^{\prime}\right\}, \\
& \pi_{\Gamma}\left(\rho_{\Gamma}^{\varepsilon_{n}}\right) \rightarrow \pi_{\Gamma}\left(\rho_{\Gamma}\right) \quad \text { strongly in } C^{0}(\bar{\Sigma}), \\
& \left(1+2 g\left(\rho^{\varepsilon_{n}}\right)\right) \partial_{t} \mu^{\varepsilon_{n}} \rightarrow(1+2 g(\rho)) \partial_{t} \mu \quad \text { weakly in } L^{2}(Q), \\
& \mu^{\varepsilon_{n}} g^{\prime}\left(\rho^{\varepsilon_{n}}\right) \partial_{t} \rho^{\varepsilon_{n}} \rightarrow \mu g^{\prime}(\rho) \partial_{t} \rho \quad \text { weakly in } L^{1}(Q), \\
& \mu^{\varepsilon_{n}} g^{\prime}\left(\rho^{\varepsilon_{n}}\right) \rightarrow \mu g^{\prime}(\rho) \quad \text { strongly in } L^{2}(Q) .
\end{aligned}
$$

Moreover, by a standard argument in the theory of maximal monotone operators (see, e. g., [1, Lemma 2.3, p. 38]), we infer that also $\rho \in D(\beta)$ and $\xi \in \beta(\rho)$ almost everywhere in $Q$, as well $\rho_{\Gamma} \in D\left(\beta_{\Gamma}\right)$ and $\xi_{\Gamma} \in \beta_{\Gamma}\left(\rho_{\Gamma}\right)$ almost everywhere on $\Sigma$. In particular, thanks to (A5), the values of $\rho$ belong almost everywhere to the domain of definition of the original function $g$. Hence, we may pass to the limit as $\varepsilon_{n} \searrow 0$ in the system (3.89)(3.93) to infer that the quintuple $\left(\mu, \rho, \rho_{\Gamma}, \xi, \xi_{\Gamma}\right)$ is a solution to the system (1.1)-(1.7).

Finally, we have that $\partial_{\mathbf{n}} \mu=0$ a.e. on $\Sigma$ and, in addition, that $g(\rho), g^{\prime}(\rho) \in C^{0}(\bar{Q})$ and $\mu \in L^{\infty}(Q)$. Since

$$
\partial_{t} \mu-(1+2 g(\rho))^{-1} \Delta \mu=-(1+2 g(\rho))^{-1} \mu g^{\prime}(\rho) \partial_{t} \rho
$$

we may view (1.1) as a linear uniformly parabolic equation for $\mu$ with continuous coefficients and a right-hand side in $L^{\infty}(0, T ; H) \cap L^{2}\left(0, T ; L^{6}(\Omega)\right)$. As $\mu_{0} \in W$, it follows from optimal $L^{p}$ - $L^{q}$-regularity results (cf. [29, Thm. 2.3]) that $\mu \in W^{1, p}(0, T ; H) \cap L^{p}(0, T ; W)$, 
for all $p \in[1,+\infty)$. We have thus shown the existence of a solution having the asserted regularity properties.

STEP 5: It remains to prove the uniqueness result. For this purpose, we follow closely the proof of [19, Thm. 3.9] for the case that $\rho$ satisfies a zero Neumann condition, pointing out the differences originating from the dynamic boundary condition (1.5). First, it is not difficult to check that Eq. (1.1) can be rewritten as

$$
\partial_{t}(\mu / \alpha(\rho))-\alpha(\rho) \Delta \mu=0 \quad \text { a.e. in } Q,
$$

where $\alpha: \overline{D(\beta)} \rightarrow(0,+\infty)$ is given by

$$
\alpha(r):=(1+2 g(r))^{-1 / 2} \text { for } r \in \overline{D(\beta)} .
$$

Now suppose that two solutions $\left(\mu_{i}, \rho_{i}, \rho_{i_{\Gamma}}, \xi_{i}, \xi_{i_{\Gamma}}\right), i=1,2$, to the system (1.1)-(1.7) with the regularity (2.5)-(2.8) are given such that $\mu_{i} \geq 0$ almost everywhere in $Q$, for $i=1,2$. We put $a_{i}:=\alpha\left(\rho_{i}\right), z_{i}:=\mu_{i} / a_{i}$, for $i=1,2$, and set

$$
\begin{aligned}
& \mu:=\mu_{1}-\mu_{2}, \quad \rho:=\rho_{1}-\rho_{2}, \quad \rho_{\Gamma}:=\rho_{1_{\Gamma}}-\rho_{2_{\Gamma}}, \quad \xi:=\xi_{1}-\xi_{2}, \quad \xi_{\Gamma}:=\xi_{1_{\Gamma}}-\xi_{2_{\Gamma}}, \\
& a:=a_{1}-a_{2}, \quad z:=z_{1}-z_{2},
\end{aligned}
$$

where we notice that $\rho_{\Gamma}=\rho_{\mid \Sigma}$ almost everywhere on $\Sigma$. Moreover, $z_{i}$ is bounded (since $\mu_{i}$ and $\rho_{i}$ are bounded), for $i=1,2$, and we have that

$$
\left|\nabla \rho_{i}\right|,\left|\nabla \mu_{i}\right|,\left|\nabla z_{i}\right| \in L^{4}\left(0, T ; L^{6}(\Omega)\right), \quad \text { for } i=1,2 .
$$

In the following estimates, we denote by $C$ or $\widehat{C}$ positive constants that may depend on the data of the system and on finite norms of the quantities $\mu_{i}, \rho_{i}, z_{i}, i=1,2$. The constants $C$ may change their meaning within formulas and even within lines.

At first, we write Eq. (3.102) for $\mu_{i}, \rho_{i}, i=1,2$, and subtract the equations from each other, which yields the identity $z_{t}-a_{1} \Delta\left(a_{1} z_{1}\right)+a_{2} \Delta\left(a_{2} z_{2}\right)=0$. Note that both $a_{1} z_{1}$ and $a_{2} z_{2}$ satisfy the Neumann homogeneous boundary condition (1.2). Then, multiplication by $z$ and integration over $Q_{t}$, where $t \in(0, T]$, leads to the equation

$$
\frac{1}{2} \int_{\Omega}|z(t)|^{2} \mathrm{~d} x+\int_{0}^{t} \int_{\Omega}\left(\nabla\left(a_{1} z\right) \cdot \nabla\left(a_{1} z_{1}\right)-\nabla\left(a_{2} z\right) \cdot \nabla\left(a_{2} z_{2}\right)\right) \mathrm{d} x \mathrm{~d} s=0,
$$

whence, arguing exactly as between the formulas (6.8) and (6.10) in the proof of $[19$, Thm. 3.9], we conclude the estimate

$$
\begin{aligned}
& \frac{1}{2} \int_{\Omega}|z(t)|^{2} \mathrm{~d} x+\frac{1}{2} \int_{0}^{t} \int_{\Omega}\left|\nabla\left(a_{1} z\right)\right|^{2} \mathrm{~d} x \mathrm{~d} s \leq(1+\widehat{C}) \int_{0}^{t} \int_{\Omega}|\nabla \rho|^{2} \mathrm{~d} x \mathrm{~d} s \\
& +C \int_{0}^{t} \int_{\Omega}\left|\nabla \mu_{2}\right|^{2}|z|^{2} \mathrm{~d} x \mathrm{~d} s+C \int_{0}^{t} \int_{\Omega}\left(\left|\nabla \rho_{1}\right|^{2}+\left|\nabla \rho_{2}\right|^{2}+\left|\nabla \mu_{2}\right|^{2}+\left|\nabla z_{2}\right|^{2}\right)|\rho|^{2} \mathrm{~d} x \mathrm{~d} s .
\end{aligned}
$$

Next, we write the equations (1.3) and (1.5) for the two solutions and subtract to obtain the identities

$$
\begin{aligned}
& \rho_{t}-\Delta \rho+\xi=\pi\left(\rho_{2}\right)-\pi\left(\rho_{1}\right)+\mu g^{\prime}\left(\rho_{1}\right)+\mu_{2}\left(g^{\prime}\left(\rho_{1}\right)-g^{\prime}\left(\rho_{2}\right)\right) \quad \text { a.e. in } Q, \\
& \partial_{\mathbf{n}} \rho+\partial_{t} \rho_{\Gamma}-\Delta_{\Gamma} \rho_{\Gamma}+\xi_{\Gamma}=\pi_{\Gamma}\left(\rho_{2_{\Gamma}}\right)-\pi_{\Gamma}\left(\rho_{1_{\Gamma}}\right) \quad \text { a.e. on } \Sigma .
\end{aligned}
$$


Thus, multiplying (3.107) by $\rho$ and integrating over $Q_{t}$, where $t \in(0, T]$, we easily derive from Young's inequality the estimate

$$
\begin{aligned}
& \frac{1}{2}\left(\|\rho(t)\|_{H}^{2}+\left\|\rho_{\Gamma}(t)\right\|_{H_{\Gamma}}^{2}\right)+\int_{0}^{t} \int_{\Omega}|\nabla \rho|^{2} \mathrm{~d} x \mathrm{~d} s+\int_{0}^{t} \int_{\Gamma}\left|\nabla_{\Gamma} \rho_{\Gamma}\right|^{2} \mathrm{~d} \Gamma \mathrm{d} s \\
& +\int_{0}^{t} \int_{\Omega} \xi \rho \mathrm{d} x \mathrm{~d} s+\int_{0}^{t} \int_{\Gamma} \xi_{\Gamma} \rho_{\Gamma} \mathrm{d} \Gamma \mathrm{d} s \\
& \leq C \int_{0}^{t} \int_{\Omega}\left(|\mu|^{2}+|\rho|^{2}\right) \mathrm{d} x \mathrm{~d} s+C \int_{0}^{t} \int_{\Gamma}\left|\rho_{\Gamma}\right|^{2} \mathrm{~d} \Gamma \mathrm{d} s,
\end{aligned}
$$

where, owing to the monotonicity of $\beta$ and $\beta_{\Gamma}$, the last two summands on the left-hand side are nonnegative. At this point, we multiply the inequality (3.109) by $2+\widehat{C}$ and add the result to (3.106). Using the estimate

$$
|\mu|=\left|a_{1} z_{1}-a_{2} z_{2}\right| \leq|a|\left|z_{1}\right|+\left|a_{2}\right||z| \leq C(|\rho|+|z|),
$$

we then easily deduce that

$$
\begin{aligned}
& \|z(t)\|_{H}^{2}+\|\rho(t)\|_{H}^{2}+\left\|\rho_{\Gamma}(t)\right\|_{H_{\Gamma}}^{2} \\
& +\int_{0}^{t} \int_{\Omega}\left(\left|\nabla\left(a_{1} z\right)\right|^{2}+|\nabla \rho|^{2}\right) \mathrm{d} x \mathrm{~d} s+\int_{0}^{t} \int_{\Gamma}\left|\nabla_{\Gamma} \rho_{\Gamma}\right|^{2} \mathrm{~d} \Gamma \mathrm{d} s \\
& \leq C \int_{0}^{t} \int_{\Omega}\left(1+\left|\nabla \rho_{1}\right|+\left|\nabla \rho_{2}\right|+\left|\nabla \mu_{2}\right|+\left|\nabla z_{2}\right|\right)^{2}\left(|z|^{2}+|\rho|^{2}\right) \mathrm{d} x \mathrm{~d} s \\
& \quad+C \int_{0}^{t} \int_{\Gamma}\left|\rho_{\Gamma}\right|^{2} \mathrm{~d} \Gamma \mathrm{d} s
\end{aligned}
$$

Now, by virtue of (3.104), the function $k:=1+\left|\nabla \rho_{1}\right|+\left|\nabla \rho_{2}\right|+\left|\nabla \mu_{2}\right|+\left|\nabla z_{2}\right|$ belongs to $L^{4}\left(0, T ; L^{6}(\Omega)\right)$. A direct application of $[19$, Lem. 6.2$]$ then yields that the first summand on the right-hand side of (3.111) is bounded by an expression of the form

$$
\begin{aligned}
& \frac{1}{2} \int_{0}^{t} \int_{\Omega}\left(\left|\nabla\left(a_{1} z\right)\right|^{2}+|\nabla \rho|^{2}\right) \mathrm{d} x \mathrm{~d} s \\
& +C \int_{0}^{t}\left(1+\left\|\nabla \rho_{1}(s)\right\|_{6}^{4}+\|k(s)\|_{6}^{4}\right)\left(\|z(s)\|_{H}^{2}+\|\rho(s)\|_{H}^{2}\right) \mathrm{d} s .
\end{aligned}
$$

Combining this with (3.111), we thus can infer from Gronwall's lemma that $z=\rho=0$ a. e. in $Q$ and $\rho_{\Gamma}=0$ a.e. on $\Sigma$. Hence, by (3.110), also $\mu=0$, and, by comparison in (3.107), $\xi=0$, a.e. in $Q$. Finally, by comparison in $(3.108), \xi_{\Gamma}=0$ a.e. on $\Sigma$. This concludes the proof of uniqueness. The assertion is thus completely shown.

REMARK 3.1: Since the expression on the right-hand side of (3.101) belongs to the space $L^{2}\left(0, T ; L^{6}(\Omega)\right)$, it follows from the optimal $L^{p}$ - $L^{q}$-regularity result of [29, Thm. 2.3]) that also $\mu \in H^{1}\left(0, T ; L^{6}(\Omega)\right) \cap L^{2}\left(0, T ; W^{2,6}(\Omega)\right)$.

\section{Stability and further regularity}

This section is devoted to the proofs of Theorem 2.3 and Theorem 2.4. 
Proof of Theorem 2.3: $\quad$ Assume that $u_{\Gamma} \in L^{\infty}(\Sigma), \beta^{\circ}\left(\rho_{0}\right) \in L^{\infty}(\Omega)$, and $\beta^{\circ}\left(\rho_{0_{\Gamma}}\right) \in$ $L^{\infty}(\Gamma)$. We need to show that the unique solution $\left(\mu, \rho, \rho_{\Gamma}, \xi, \xi_{\Gamma}\right)$ has the additional property that $\xi \in L^{\infty}(Q)$. To this end, we recall that the approximating system (3.89)(3.93) introduced in Step 3 of the proof of Theorem 2.1 has a solution triple $\left(\mu^{\varepsilon}, \rho^{\varepsilon}, \rho_{\Gamma}^{\varepsilon}\right)$ satisfying the estimate (3.94). Moreover, $\left\{\rho^{\varepsilon}\right\}$ is bounded in $C^{0}(\bar{Q})$ uniformly with respect to $\varepsilon>0$, which entails, in particular, that $\beta^{\varepsilon}\left(\rho^{\varepsilon}\right) \in C^{0}(\bar{Q})$, and thus $\beta^{\varepsilon}\left(\rho_{\Gamma}^{\varepsilon}\right) \in$ $C^{0}(\bar{\Sigma})$ and $\beta_{\Gamma}^{\varepsilon}\left(\rho_{\Gamma}^{\varepsilon}\right) \in C^{0}(\bar{\Sigma})$. We therefore may multiply $(3.91)$ by $\left(\beta^{\varepsilon}\left(\rho^{\varepsilon}\right)\right)^{2 k-1}$, for every fixed $k \in \mathbb{N}$. Integration over $Q_{t}$, where $0<t \leq T$, yields the identity

$$
\begin{aligned}
& \int_{\Omega} \Phi^{\varepsilon}(x, t) \mathrm{d} x+\int_{\Gamma} \Phi_{\Gamma}^{\varepsilon}(x, t) \mathrm{d} \Gamma+(2 k-1) \int_{0}^{t} \int_{\Omega}\left|\beta^{\varepsilon}\left(\rho^{\varepsilon}\right)\right|^{2 k-2}\left(\beta^{\varepsilon}\right)^{\prime}\left(\rho^{\varepsilon}\right)\left|\nabla \rho^{\varepsilon}\right|^{2} \mathrm{~d} x \mathrm{~d} s \\
& +(2 k-1) \int_{0}^{t} \int_{\Gamma}\left|\beta^{\varepsilon}\left(\rho_{\Gamma}^{\varepsilon}\right)\right|^{2 k-2}\left(\beta^{\varepsilon}\right)^{\prime}\left(\rho_{\Gamma}^{\varepsilon}\right)\left|\nabla_{\Gamma} \rho_{\Gamma}^{\varepsilon}\right|^{2} \mathrm{~d} \Gamma \mathrm{d} s+\int_{0}^{t} \int_{\Omega}\left|\beta^{\varepsilon}\left(\rho^{\varepsilon}\right)\right|^{2 k} \mathrm{~d} x \mathrm{~d} s \\
& =\int_{\Omega} \Phi^{\varepsilon}(x, 0) \mathrm{d} x+\int_{\Gamma} \Phi_{\Gamma}^{\varepsilon}(x, 0) \mathrm{d} \Gamma+\int_{0}^{t} \int_{\Omega}\left(\beta^{\varepsilon}\left(\rho^{\varepsilon}\right)\right)^{2 k-1}\left(\mu^{\varepsilon} g^{\prime}\left(\rho^{\varepsilon}\right)-\pi\left(\rho^{\varepsilon}\right)\right) \mathrm{d} x \mathrm{~d} s \\
& \quad+\int_{0}^{t} \int_{\Gamma}\left(\beta^{\varepsilon}\left(\rho_{\Gamma}^{\varepsilon}\right)\right)^{2 k-1}\left(-\beta_{\Gamma}^{\varepsilon}\left(\rho_{\Gamma}^{\varepsilon}\right)-\pi_{\Gamma}\left(\rho_{\Gamma}^{\varepsilon}\right)+u_{\Gamma}\right) \mathrm{d} \Gamma \mathrm{d} s
\end{aligned}
$$

with the functions

$$
\Phi^{\varepsilon}(x, t):=\int_{0}^{\rho^{\varepsilon}(x, t)}\left(\beta^{\varepsilon}(r)\right)^{2 k-1} \mathrm{~d} r, \quad \Phi_{\Gamma}^{\varepsilon}(x, t):=\int_{0}^{\rho_{\Gamma}^{\varepsilon}(x, t)}\left(\beta^{\varepsilon}(r)\right)^{2 k-1} \mathrm{~d} r .
$$

Since the functions $\beta^{\varepsilon}$ and $\beta_{\Gamma}^{\varepsilon}$ vanish at zero and are monotone, all of the expressions on the left-hand side are nonnegative. We estimate the terms of the right-hand side individually, where we denote by $C_{i}, i \in \mathbb{N}$, positive constants that may depend on the data of the system but not on $\varepsilon>0$. At first, invoking (A1), (2.1), and the assumption (2.9) that implies $\beta^{\circ}\left(\rho_{0}\right) \in L^{\infty}(\Omega)$, we obtain for the first summand on the right-hand side the estimate

$$
\begin{aligned}
& \int_{\Omega} \int_{0}^{\rho_{0}(x)}\left(\beta^{\varepsilon}(r)\right)^{2 k-1} \mathrm{~d} r \mathrm{~d} x \leq \int_{\Omega}\left|\rho_{0}(x)\right|\left|\beta^{\varepsilon}\left(\rho_{0}(x)\right)\right|^{2 k-1} \mathrm{~d} x \\
& \leq\left\|\rho_{0}\right\|_{\infty} \int_{\Omega}\left|\beta^{\circ}\left(\rho_{0}(x)\right)\right|^{2 k-1} \mathrm{~d} x \leq C_{1}\left\|\beta^{\circ}\left(\rho_{0}\right)\right\|_{\infty}^{2 k-1} \leq C_{2} C_{3}^{2 k} .
\end{aligned}
$$

By the same token, we have that

$$
\int_{\Gamma} \Phi_{\Gamma}^{\varepsilon}(x, 0) \mathrm{d} x \leq C_{4} C_{5}^{2 k}
$$

Next, recall that $\left\|\mu^{\varepsilon} g^{\prime}\left(\rho^{\varepsilon}\right)-\pi\left(\rho^{\varepsilon}\right)\right\|_{L^{\infty}(Q)} \leq C_{6}$. Hence, using Young's inequality (1.23) with $p=\frac{2 k}{2 k-1}, q=2 k$, and $\gamma=\left(\frac{p}{2}\right)^{1 / p}$, we obtain for the third integral on the right-hand side, which we denote by $I_{1}$, the following estimate:

$$
\begin{aligned}
& I_{1} \leq C_{6} \int_{0}^{t} \int_{\Omega}\left|\beta^{\varepsilon}\left(\rho^{\varepsilon}\right)\right|^{2 k-1} \mathrm{~d} x \mathrm{~d} s \leq \frac{1}{2} \int_{0}^{t} \int_{\Omega}\left|\beta^{\varepsilon}\left(\rho^{\varepsilon}\right)\right|^{2 k} \mathrm{~d} x \mathrm{~d} s+\frac{1}{2 k}\left(\frac{2 k-1}{k}\right)^{2 k-1} C_{6}^{2 k} \\
& \leq \frac{1}{2} \int_{0}^{t} \int_{\Omega}\left|\beta^{\varepsilon}\left(\rho^{\varepsilon}\right)\right|^{2 k} \mathrm{~d} x \mathrm{~d} s+C_{7} C_{8}^{2 k}
\end{aligned}
$$


Finally, since $u_{\Gamma} \in L^{\infty}(\Sigma)$ by assumption, we obtain that $\left\|u_{\Gamma}-\pi_{\Gamma}\left(\rho_{\Gamma}\right)\right\|_{L^{\infty}(\Sigma)} \leq C_{9}$. Moreover, we have, using (3.1), and invoking Young's inequality similarly as above,

$$
\begin{aligned}
& -\left(\beta^{\varepsilon}\left(\rho_{\Gamma}^{\varepsilon}\right)\right)^{2 k-1} \beta_{\Gamma}^{\varepsilon}\left(\rho_{\Gamma}^{\varepsilon}\right)=-\left|\beta^{\varepsilon}\left(\rho_{\Gamma}^{\varepsilon}\right)\right|^{2 k-1}\left|\beta_{\Gamma}^{\varepsilon}\left(\rho_{\Gamma}^{\varepsilon}\right)\right| \leq-\frac{1}{\eta}\left|\beta^{\varepsilon}\left(\rho_{\Gamma}^{\varepsilon}\right)\right|^{2 k}+\frac{C_{\Gamma}}{\eta}\left|\beta^{\varepsilon}\left(\rho_{\Gamma}^{\varepsilon}\right)\right|^{2 k-1} \\
& \leq-\frac{1}{2 \eta}\left|\beta^{\varepsilon}\left(\rho_{\Gamma}^{\varepsilon}\right)\right|^{2 k}+C_{10} C_{11}^{2 k}
\end{aligned}
$$

Hence, the last integral on the right-hand side of (4.1), which we denote by $I_{2}$, admits an estimate of the form

$$
I_{2} \leq-\frac{1}{2 \eta} \int_{0}^{t} \int_{\Gamma}\left|\beta^{\varepsilon}\left(\rho_{\Gamma}^{\varepsilon}\right)\right|^{2 k} \mathrm{~d} \Gamma \mathrm{d} s+C_{12} C_{13}^{2 k} .
$$

Combining the above estimates, we have thus shown that

$$
\left\|\beta^{\varepsilon}\left(\rho^{\varepsilon}\right)\right\|_{L^{2 k}(Q)}^{2 k}+\left\|\beta^{\varepsilon}\left(\rho_{\Gamma}^{\varepsilon}\right)\right\|_{L^{2 k}(\Sigma)}^{2 k} \leq C_{14} C_{15}^{2 k} \quad \text { for all } k \in \mathbb{N} .
$$

Taking the $(2 k)$-th root on both sides of this inequality and then letting $k \rightarrow \infty$, we conclude that

$$
\left\|\beta^{\varepsilon}\left(\rho^{\varepsilon}\right)\right\|_{L^{\infty}(Q)}+\left\|\beta^{\varepsilon}\left(\rho_{\Gamma}^{\varepsilon}\right)\right\|_{L^{\infty}(\Sigma)} \leq C_{16} .
$$

But this means that the convergence result (3.98) in Step 4 of the proof of Theorem 2.1 can be replaced by the stronger statement

$$
\beta^{\varepsilon_{n}}\left(\rho^{\varepsilon_{n}}\right) \rightarrow \xi \quad \text { weakly-star in } L^{\infty}(Q),
$$

so that the solution constructed there satisfies $\xi \in L^{\infty}(Q)$. Since the solution is unique, the assertion is proved.

Before proving Theorem 2.4, we cite a known auxiliary result (cf. [26, Thm. 2.2]) that will be used during the course of the proof.

LEMmA 4.1: Suppose that functions $a \in L^{\infty}(Q), a_{\Gamma} \in L^{\infty}(\Sigma), \sigma \in L^{2}(Q)$, and $\sigma_{\Gamma} \in L^{2}(\Sigma)$ are given. Then the linear initial-boundary value problem

$$
\begin{aligned}
& \partial_{t} y-\Delta y+a y=\sigma \quad \text { a.e. in } Q, \\
& \partial_{\mathbf{n}} y+\partial_{t} y_{\Gamma}-\Delta_{\Gamma} y_{\Gamma}+a_{\Gamma} y_{\Gamma}=\sigma_{\Gamma}, \quad y_{\Gamma}=y_{\mid \Sigma}, \quad \text { a.e. on } \Sigma, \\
& y(0)=0 \quad \text { a.e. in } \Omega, \quad y_{\Gamma}(0)=0 \quad \text { a.e. on } \Gamma,
\end{aligned}
$$

has a unique solution pair satisfying $y \in H^{1}(0, T ; H) \cap C^{0}([0, T] ; V) \cap L^{2}\left(0, T ; H^{2}(\Omega)\right)$ and $y_{\Gamma} \in H^{1}\left(0, T ; H_{\Gamma}\right) \cap C^{0}\left([0, T] ; V_{\Gamma}\right) \cap L^{2}\left(0, T ; H^{2}(\Gamma)\right)$. Moreover, there is a constant $C_{L}>0$, which depends only on $\Omega, T,\|a\|_{L^{\infty}(Q)}$, and $\left\|a_{\Gamma}\right\|_{L^{\infty}(\Sigma)}$, such that, for every $t \in(0, T]$

$$
\begin{aligned}
& \|y\|_{H^{1}(0, t ; H) \cap C^{0}([0, t] ; V) \cap L^{2}\left(0, t ; H^{2}(\Omega)\right)}+\left\|y_{\Gamma}\right\|_{H^{1}\left(0, t ; H_{\Gamma}\right) \cap C^{0}\left([0, t] ; V_{\Gamma}\right) \cap L^{2}\left(0, t ; H^{2}(\Gamma)\right)} \\
& \leq C_{L}\left(\|\sigma\|_{L^{2}\left(Q_{t}\right)}+\left\|\sigma_{\Gamma}\right\|_{L^{2}\left(\Sigma_{t}\right)}\right) .
\end{aligned}
$$

We are now prepared to prove Theorem 2.4. 
Proof of Theorem 2.4: We first prove (2.10) by recalling that both $\rho$ and $\xi$ are bounded (see Rem. 2.2 and Thm. 2.3). We construct $r^{*}$. If $r_{+}=+\infty$ we can take $r^{*}:=\sup \rho<r_{+}$. If instead $r_{+}<+\infty$, we choose $s^{*} \geq 0$ such that $s^{*} \geq \xi$ a.e. in $Q$. By observing that $\lim _{r} \gamma_{+} \beta^{\circ}(r)=+\infty$ by maximal monotonicity, we find $r^{*} \in\left(0, r_{+}\right)$ such that $\beta^{\circ}(r)>s^{*}$ for every $r \in\left(r^{*}, r_{+}\right)$. Then, it holds that $\rho \leq r^{*}$ on $\bar{Q}$. Indeed, if the continuous function $\rho$ satisfies $\rho(x, t)=r^{*}+2 \delta$ for some $\delta>0$ at some point $(x, t)$, we should have $\rho \geq r^{*}+\delta$ in some subset $Q^{\prime} \subset Q$ with positive measure. This implies that $\xi \geq \beta^{\circ}\left(r^{*}+\delta\right)>s^{*}$ a.e. in $Q^{\prime}$, and this contradicts the definition of $s^{*}$. As $r_{*}$ can be obtained similarly, the separation property (2.10) is established. From this and $D\left(\beta_{\Gamma}\right)=\left(r_{-}, r_{+}\right)$it immediately follows that even $\xi_{\Gamma}$ is bounded.

We assume that the potentials are smooth and show the validity of (2.11). To this end, suppose that $u_{i_{\Gamma}} \in H^{1}\left(0, T ; H_{\Gamma}\right) \cap L^{\infty}(\Sigma), i=1,2$, are given, and let $\left(\mu_{i}, \rho_{i}, \rho_{i_{\Gamma}}, \xi_{i}, \xi_{i_{\Gamma}}\right)$, where $\xi_{i}=\beta\left(\rho_{i}\right)$ and $\xi_{i_{\Gamma}}=\beta_{\Gamma}\left(\rho_{i_{\Gamma}}\right)$, be corresponding solutions to (1.1)-(1.7), for $i=1,2$, that enjoy the regularity properties (2.5)-(2.8). In the following, we denote by $C>0$ constants that depend only on the data of the system, on $\left\|u_{i_{\Gamma}}\right\|_{H^{1}\left(0, T ; H_{\Gamma}\right)}, i=1,2$, and on the norms of $\left(\mu_{i}, \rho_{i}, \rho_{i_{\Gamma}}\right), i=1,2$, in the spaces indicated in $(2.5)-(2.7)$. Now, we put

$$
\mu:=\mu_{1}-\mu_{2}, \quad \rho:=\rho_{1}-\rho_{2}, \quad \rho_{\Gamma}:=\rho_{1_{\Gamma}}-\rho_{2_{\Gamma}}, \quad u_{\Gamma}:=u_{1_{\Gamma}}-u_{2_{\Gamma}} .
$$

Obviously, $\rho_{\Gamma}=\rho_{\mid \Sigma}$ on $\Sigma$. Moreover, both $\rho_{i}$ and $\rho_{i_{\Gamma}}$ satisfy (2.10) for $i=1,2$. As all the functions $g, g^{\prime}, \pi, \beta, \pi_{\Gamma}$ and $\beta_{\Gamma}$ are Lipschitz continuous on $\left[r_{*}, r^{*}\right]$, we have that

$$
\begin{aligned}
& \max _{0 \leq i \leq 1}\left|g^{(i)}\left(\rho_{1}\right)-g^{(i)}\left(\rho_{2}\right)\right|+\left|\pi\left(\rho_{1}\right)-\pi\left(\rho_{2}\right)\right|+\left|\beta\left(\rho_{1}\right)-\beta\left(\rho_{2}\right)\right| \leq C|\rho| \text { in } \bar{Q} \\
& \left|\beta_{\Gamma}\left(\rho_{1_{\Gamma}}\right)-\beta_{\Gamma}\left(\rho_{2_{\Gamma}}\right)\right|+\left|\pi_{\Gamma}\left(\rho_{1_{\Gamma}}\right)-\pi_{\Gamma}\left(\rho_{2_{\Gamma}}\right)\right| \leq C\left|\rho_{\Gamma}\right| \text { on } \bar{\Sigma} .
\end{aligned}
$$

Furthermore, we easily verify that

$$
\begin{aligned}
& \left(1+2 g\left(\rho_{1}\right)\right) \partial_{t} \mu+g^{\prime}\left(\rho_{1}\right) \partial_{t} \rho_{1} \mu-\Delta=-2 \partial_{t} \mu_{2}\left(g\left(\rho_{1}\right)-g\left(\rho_{2}\right)\right) \mu \\
& -\mu_{2}\left(g^{\prime}\left(\rho_{1}\right)-g^{\prime}\left(\rho_{2}\right)\right) \partial_{t} \rho_{1}-\mu_{2} g^{\prime}\left(\rho_{2}\right) \partial_{t} \rho \text { a.e. in } Q \text {, } \\
& \partial_{\mathbf{n}} \mu=0 \quad \text { a.e. on } \Sigma, \quad \mu(0)=0 \text { a.e. in } \Omega \text {, } \\
& \partial_{t} \rho-\Delta \rho=\mu_{1}\left(g^{\prime}\left(\rho_{1}\right)-g^{\prime}\left(\rho_{2}\right)\right)+g^{\prime}\left(\rho_{2}\right) \mu+\pi\left(\rho_{2}\right)-\pi\left(\rho_{1}\right) \\
& +\beta\left(\rho_{2}\right)-\beta\left(\rho_{1}\right) \text { a.e. in } Q \text {, } \\
& \partial_{\mathbf{n}} \rho+\partial_{t} \rho_{\Gamma}-\Delta_{\Gamma} \rho_{\Gamma}=\beta_{\Gamma}\left(\rho_{2_{\Gamma}}\right)-\beta_{\Gamma}\left(\rho_{1_{\Gamma}}\right)+\pi_{\Gamma}\left(\rho_{2_{\Gamma}}\right)-\pi_{\Gamma}\left(\rho_{1_{\Gamma}}\right)+u_{\Gamma} \quad \text { a.e. on } \Sigma \text {, } \\
& \rho(0)=0 \quad \text { a.e. in } \Omega, \rho_{\Gamma}(0)=0 \quad \text { a.e. on } \Gamma \text {. }
\end{aligned}
$$

First, we observe that $\partial_{t}\left(\left(\frac{1}{2}+g\left(\rho_{1}\right)\right) \mu^{2}\right)=\left(1+2 g\left(\rho_{1}\right)\right) \mu \partial_{t} \mu+g^{\prime}\left(\rho_{1}\right) \partial_{t} \rho_{1} \mu^{2}$. Hence, if we multiply (4.17) by $\mu$ and integrate over $Q_{t}$, where $t \in(0, T]$, then we obtain the estimate

$$
\int_{\Omega}\left(\frac{1}{2}+g\left(\rho_{1}(t)\right)\right)|\mu(t)|^{2} \mathrm{~d} x+\int_{0}^{t} \int_{\Omega}|\nabla \mu|^{2} \mathrm{~d} x \mathrm{~d} s \leq I_{1}+I_{2}+I_{3},
$$

where the expressions $I_{j}, 1 \leq j \leq 3$, are defined and estimated as follows: at first, we use Hölder's and Young's inequalities, the continuity of the embedding $V \subset L^{4}(\Omega)$, and 
(4.15), to obtain that

$$
\begin{aligned}
I_{1} & =-\int_{0}^{t} \int_{\Omega} 2 \partial_{t} \mu_{2}\left(g\left(\rho_{1}\right)-g\left(\rho_{2}\right)\right) \mu \mathrm{d} x \mathrm{~d} s \leq C \int_{0}^{t}\left\|\partial_{t} \mu_{2}(s)\right\|_{2}\|\rho(s)\|_{4}\|\mu(s)\|_{4} \mathrm{~d} s \\
& \leq \frac{1}{2} \int_{0}^{t}\|\mu(s)\|_{V}^{2} \mathrm{~d} s+C \int_{0}^{t}\left\|\partial_{t} \mu_{2}(s)\right\|_{H}^{2}\|\rho(s)\|_{V}^{2} \mathrm{~d} s .
\end{aligned}
$$

By the same token, we infer that

$$
\begin{aligned}
I_{2} & =-\int_{0}^{t} \int_{\Omega} \mu_{2}\left(g^{\prime}\left(\rho_{1}\right)-g^{\prime}\left(\rho_{2}\right)\right) \partial_{t} \rho_{1} \mu \mathrm{d} x \mathrm{~d} s \leq C \int_{0}^{t}\left\|\partial_{t} \rho_{1}(s)\right\|_{4}\|\rho(s)\|_{4}\|\mu(s)\|_{2} \mathrm{~d} s \\
& \leq C \int_{0}^{t} \int_{\Omega}|\mu|^{2} \mathrm{~d} x \mathrm{~d} s+C \int_{0}^{t}\left\|\partial_{t} \rho_{1}(s)\right\|_{V}^{2}\|\rho(s)\|_{V}^{2} \mathrm{~d} s .
\end{aligned}
$$

Finally, we have that

$$
I_{3}=-\int_{0}^{t} \int_{\Omega} \mu_{2} g^{\prime}\left(\rho_{2}\right) \partial_{t} \rho \mu \mathrm{d} x \mathrm{~d} s \leq \frac{1}{4} \int_{0}^{t} \int_{\Omega}\left|\partial_{t} \rho\right|^{2} \mathrm{~d} x \mathrm{~d} s+C \int_{0}^{t} \int_{\Omega}|\mu|^{2} \mathrm{~d} x \mathrm{~d} s .
$$

Combining (4.22)-(4.25), and recalling that $g$ is nonnegative, we have thus shown an estimate of the form

$$
\begin{aligned}
& \frac{1}{2} \int_{\Omega}|\mu(t)|^{2} \mathrm{~d} x+\frac{1}{2} \int_{0}^{t}\|\mu(s)\|_{V}^{2} \mathrm{~d} s \leq \frac{1}{4} \int_{0}^{t} \int_{\Omega}\left|\partial_{t} \rho\right|^{2} \mathrm{~d} x \mathrm{~d} s \\
& \quad+C \int_{0}^{t}\left(1+\left\|\partial_{t} \mu_{2}(s)\right\|_{H}^{2}+\left\|\partial_{t} \rho_{1}(s)\right\|_{V}^{2}\right)\left(\|\rho(s)\|_{H}^{2}+\|\mu(s)\|_{H}^{2}\right) \mathrm{d} s .
\end{aligned}
$$

Now, we add $\rho$ to both sides of (4.19) and $\rho_{\Gamma}$ to both sides of (4.20). Then we multiply (4.17) by $\partial_{t} \rho$ and integrate over $Q_{t}$, where $0<t \leq T$. Using (4.15) and (4.16), we deduce that

$$
\begin{aligned}
& \int_{0}^{t} \int_{\Omega}\left|\partial_{t} \rho\right|^{2} \mathrm{~d} x \mathrm{~d} s+\int_{0}^{t} \int_{\Gamma}\left|\partial_{t} \rho_{\Gamma}\right|^{2} \mathrm{~d} \Gamma \mathrm{d} s+\frac{1}{2}\left(\|\rho(t)\|_{V}^{2}+\left\|\rho_{\Gamma}(s)\right\|_{V_{\Gamma}}^{2}\right) \\
& \leq C \int_{0}^{t} \int_{\Omega}\left|\partial_{t} \rho\right|(|\mu|+|\rho|) \mathrm{d} x \mathrm{~d} s+C \int_{0}^{t} \int_{\Gamma}\left|\partial_{t} \rho_{\Gamma}\right|\left(\left|\rho_{\Gamma}\right|+\left|u_{\Gamma}\right|\right) \mathrm{d} \Gamma \mathrm{d} s \\
& \leq \frac{1}{4} \int_{0}^{t} \int_{\Omega}\left|\partial_{t} \rho\right|^{2} \mathrm{~d} x \mathrm{~d} s+\frac{1}{2} \int_{0}^{t} \int_{\Gamma}\left|\rho_{\Gamma}\right|^{2} \mathrm{~d} x \mathrm{~d} s+C \int_{0}^{t} \int_{\Gamma}\left|u_{\Gamma}\right|^{2} \mathrm{~d} \Gamma \mathrm{d} s \\
& \quad+C \int_{0}^{t} \int_{\Omega}\left(|\rho|^{2}+|\mu|^{2}\right) \mathrm{d} x \mathrm{~d} s+C \int_{0}^{t} \int_{\Gamma}\left|\rho_{\Gamma}\right|^{2} \mathrm{~d} \Gamma \mathrm{d} s
\end{aligned}
$$

Now, we add (4.26) to the final result of (4.27). Observe that the mapping $s \mapsto$ $\left\|\partial_{t} \mu_{2}(s)\right\|_{H}^{2}+\left\|\partial_{t} \rho_{1}(s)\right\|_{V}^{2}$ is known to belong to $L^{1}(0, T)$. Hence, invoking Gronwall's lemma, we can infer from (4.26) and (4.27) the estimate

$$
\begin{aligned}
& \|\mu\|_{L^{\infty}(0, t ; H) \cap L^{2}(0, t ; V)}+\|\rho\|_{H^{1}(0, t ; H) \cap L^{\infty}(0, t ; V)} \\
& \quad+\left\|\rho_{\Gamma}\right\|_{H^{1}\left(0, t ; H_{\Gamma}\right) \cap L^{\infty}\left(0, t ; V_{\Gamma}\right)} \leq C\left\|u_{\Gamma}\right\|_{L^{2}\left(0, t ; H_{\Gamma}\right)} .
\end{aligned}
$$

At this point, we observe that the system (4.19)-(4.21) is of the form (4.11)-(4.13), with $y:=\rho, y_{\Gamma}:=\rho_{\Gamma}, a:=1, a_{\Gamma}:=1$, and where $\sigma$ and $\sigma_{\Gamma}$ are given by the right-hand 
sides of (4.19) and (4.20), respectively. Therefore, invoking (4.15), (4.16), and (4.28), we conclude from Lemma 4.1 that

$$
\begin{aligned}
& \|\rho\|_{H^{1}(0, t ; H) \cap C^{0}([0, t] ; V) \cap L^{2}\left(0, t ; H^{2}(\Omega)\right)}+\left\|\rho_{\Gamma}\right\|_{H^{1}\left(0, t ; H_{\Gamma}\right) \cap C^{0}\left([0, t] ; V_{\Gamma}\right) \cap L^{2}\left(0, t ; H^{2}(\Gamma)\right)} \\
& \quad \leq C\left\|u_{\Gamma}\right\|_{L^{2}\left(0, t ; H_{\Gamma}\right)} .
\end{aligned}
$$

With this, the stability estimate (2.11) is shown.

\section{References}

[1] V. Barbu, "Nonlinear differential equations of monotone types in Banach spaces", Springer, New York, 2010.

[2] H. Brézis, "Opérateurs maximaux monotones et semi-groupes de contractions dans les espaces de Hilbert", North-Holland Math. Stud. 5, North-Holland, Amsterdam, 1973.

[3] F. Brezzi, G. Gilardi, Chapters 1-3 in "Finite Element Handbook", H. Kardestuncer and D.H. Norrie (eds.), McGraw-Hill Book Co., New York, 1987.

[4] J.W. Cahn, J.E. Hilliard, Free energy of a nonuniform system I. Interfacial free energy, J. Chem. Phys. 2 (1958), 258-267.

[5] L. Calatroni, P. Colli, Global solution to the Allen-Cahn equation with singular potentials and dynamic boundary conditions, Nonlinear Anal. 79 (2013), 12-27.

[6] C. Cavaterra, M. Grasselli, H. Wu, Non-isothermal viscous Cahn-Hilliard equation with inertial term and dynamic boundary conditions, Commun. Pure Appl. Anal. 13 (2014), $1855-1890$.

[7] L. Cherfils, S. Gatti, A. Miranville, A variational approach to a Cahn-Hilliard model in a domain with nonpermeable walls, J. Math. Sci. (N.Y.) 189 (2013), 604-636.

[8] P. Colli, T. Fukao, The Allen-Cahn equation with dynamic boundary conditions and mass constraints, Math. Methods Appl. Sci. 38 (2015), 3950-3967.

[9] P. Colli, T. Fukao, Cahn-Hilliard equation with dynamic boundary conditions and mass constraint on the boundary, J. Math. Anal. Appl. 429 (2015), 1190-1213.

[10] P. Colli, T. Fukao, Equation and dynamic boundary condition of Cahn-Hilliard type with singular potentials, Nonlinear Anal. 127 (2015), 413-433.

[11] P. Colli, G. Gilardi, P. Krejčí, P. Podio-Guidugli, J. Sprekels, Analysis of a time discretization scheme for a nonstandard viscous Cahn-Hilliard system, ESAIM Math. Model. Numer. Anal. 48 (2014), 1061-1087.

[12] P. Colli, G. Gilardi, P. Krejčí, J. Sprekels, A vanishing diffusion limit in a nonstandard system of phase field equations, Evol. Equ. Control Theory 3 (2014), 257-275.

[13] P. Colli, G. Gilardi, P. Krejčí, J. Sprekels, A continuous dependence result for a nonstandard system of phase field equations, Math. Methods Appl. Sci. 37 (2014), 1318-1324.

[14] P. Colli, G. Gilardi, P. Podio-Guidugli, J. Sprekels, Well-posedness and long-time behavior for a nonstandard viscous Cahn-Hilliard system, SIAM J. Appl. Math. 71 (2011), 18491870 . 
[15] P. Colli, G. Gilardi, P. Podio-Guidugli, J. Sprekels, Global existence for a strongly coupled Cahn-Hilliard system with viscosity, Boll. Unione Mat. Ital. 5 (2012), 495-513.

[16] P. Colli, G. Gilardi, P. Podio-Guidugli, J. Sprekels, Distributed optimal control of a nonstandard system of phase field equations, Contin. Mech. Thermodyn. 24 (2012), 437-459.

[17] P. Colli, G. Gilardi, P. Podio-Guidugli, J. Sprekels, Continuous dependence for a nonstandard Cahn-Hilliard system with nonlinear atom mobility, Rend. Sem. Mat. Univ. Politec. Torino 70 (2012), 27-52.

[18] P. Colli, G. Gilardi, P. Podio-Guidugli, J. Sprekels, An asymptotic analysis for a nonstandard Cahn-Hilliard system with viscosity, Discrete Contin. Dyn. Syst. Ser. S 6 (2013), 353-368.

[19] P. Colli, G. Gilardi, P. Podio-Guidugli, J. Sprekels, Global existence and uniqueness for a singular/degenerate Cahn-Hilliard system with viscosity, J. Differential Equations 254 (2013), 4217-4244.

[20] P. Colli, G. Gilardi, J. Sprekels, Analysis and optimal boundary control of a nonstandard system of phase field equations, Milan J. Math. 80 (2012), 119-149.

[21] P. Colli, G. Gilardi, J. Sprekels, Regularity of the solution to a nonstandard system of phase field equations, Rend. Cl. Sci. Mat. Nat. 147 (2013), 3-19.

[22] P. Colli, G. Gilardi, J. Sprekels, On the Cahn-Hilliard equation with dynamic boundary conditions and a dominating boundary potential, J. Math. Anal. Appl. 419 (2014), 972994.

[23] P. Colli, G. Gilardi, J. Sprekels, A boundary control problem for the pure Cahn-Hilliard equation with dynamic boundary conditions, Adv. Nonlinear Anal. 4 (2015), 311-325.

[24] P. Colli, G. Gilardi, J. Sprekels, A boundary control problem for the viscous Cahn-Hilliard equation with dynamic boundary conditions, Appl. Math. Optim. 73 (2016), 195-225.

[25] P. Colli, G. Gilardi, J. Sprekels, On an application of Tikhonov's fixed point theorem to a nonlocal Cahn-Hilliard type system modeling phase separation, J. Differ. Equations 260 (2016), 7940-7964.

[26] P. Colli, J. Sprekels, Optimal control of an Allen-Cahn equation with singular potentials and dynamic boundary condition, SIAM J. Control Optim. 53 (2015), 213-234.

[27] M. Conti, S. Gatti, A. Miranville, Attractors for a Caginalp model with a logarithmic potential and coupled dynamic boundary conditions, Anal. Appl. (Singap.) 11 (2013), 1350024, $31 \mathrm{pp}$.

[28] M. Conti, S. Gatti, A. Miranville, Multi-component Cahn-Hilliard systems with dynamic boundary conditions, Nonlinear Anal. Real World Appl. 25 (2015), 137-166.

[29] R. Denk, M. Hieber, J. Prüss, Optimal $L^{p}-L^{q}$-estimates for parabolic boundary value problems with inhomogeneous data, Math. Z. 257 (2007), 193-224.

[30] C.M. Elliott, S. Zheng, On the Cahn-Hilliard equation, Arch. Rational Mech. Anal. 96 (1986), 339-357.

[31] E. Fried, M.E. Gurtin, Continuum theory of thermally induced phase transitions based on an order parameter, Phys. D 68 (1993), 326-343. 
[32] C. G. Gal, M. Grasselli, The non-isothermal Allen-Cahn equation with dynamic boundary conditions, Discrete Contin. Dyn. Syst. 22 (2008), 1009-1040.

[33] C.G. Gal, M. Warma, Well posedness and the global attractor of some quasi-linear parabolic equations with nonlinear dynamic boundary conditions, Differential Integral Equations 23 (2010), 327-358.

[34] G. Gilardi, A. Miranville, G. Schimperna, On the Cahn-Hilliard equation with irregular potentials and dynamic boundary conditions, Commun. Pure. Appl. Anal. 8 (2009), 881912.

[35] G. Gilardi, A. Miranville, G. Schimperna, Long-time behavior of the Cahn-Hilliard equation with irregular potentials and dynamic boundary conditions, Chin. Ann. Math. Ser. B 31 (2010), 679-712.

[36] G.R. Goldstein, A. Miranville, A Cahn-Hilliard-Gurtin model with dynamic boundary conditions, Discrete Contin. Dyn. Syst. Ser. S 6 (2013), 387-400.

[37] G.R. Goldstein, A. Miranville, G. Schimperna, A Cahn-Hilliard model in a domain with non-permeable walls, Phys. D 240 (2011), 754-766.

[38] M.E. Gurtin, Generalized Ginzburg-Landau and Cahn-Hilliard equations based on a microforce balance, Phys. D 92 (1996), 178-192.

[39] M. Heida, Existence of solutions for two types of generalized versions of the Cahn-Hilliard equation, Appl. Math. 60 (2015), 51-90.

[40] H. Israel, Long time behavior of an Allen-Cahn type equation with a singular potential and dynamic boundary conditions, J. Appl. Anal. Comput. 2 (2012), 29-56.

[41] M. Liero, Passing from bulk to bulk-surface evolution in the Allen-Cahn equation, NoDEA Nonlinear Differential Equations Appl. 20 (2013), 919-942.

[42] A. Miranville, E. Rocca, G. Schimperna, A. Segatti, The Penrose-Fife phase-field model with coupled dynamic boundary conditions, Discrete Contin. Dyn. Syst. 34 (2014), 42594290 .

[43] A. Novick-Cohen, On the viscous Cahn-Hilliard equation, in "Material instabilities in continuum mechanics (Edinburgh, 1985-1986)", Oxford Sci. Publ., Oxford Univ. Press, New York, 1988, pp. 329-342.

[44] P. Podio-Guidugli, Models of phase segregation and diffusion of atomic species on a lattice, Ric. Mat. 55 (2006), 105-118.

[45] J. Simon, Compact sets in the space $L^{p}(0, T ; B)$, Ann. Mat. Pura. Appl. (4) 146 (1987), 65-96. 